\title{
A VLT/FLAMES STUDY OF THE PECULIAR INTERMEDIATE-AGE LARGE MAGELLANIC CLOUD STAR CLUSTER NGC 1846. I. KINEMATICS*
}

\author{
A. D. MaCKey ${ }^{1}$, G. S. Da $\operatorname{Costa}^{1}$, A. M. N. Ferguson ${ }^{2}$, and D. Yong ${ }^{1}$ \\ ${ }^{1}$ Research School of Astronomy \& Astrophysics, The Australian National University, Mount Stromlo Observatory, \\ via Cotter Road, Weston, ACT 2611, Australia; dougal@mso.anu.edu.au \\ 2 Institute for Astronomy, University of Edinburgh, Royal Observatory, Blackford Hill, Edinburgh, EH9 3HJ, UK \\ Received 2012 August 22; accepted 2012 November 12; published 2012 December 14
}

\begin{abstract}
In this paper we present high-resolution VLT/FLAMES observations of red giant stars in the massive intermediateage Large Magellanic Cloud star cluster NGC 1846, which, on the basis of its extended main-sequence turnoff (EMSTO), possesses an internal age spread of $\approx 300$ Myr. We describe in detail our target selection and data reduction procedures, and construct a sample of 21 stars possessing radial velocities indicating their membership of NGC 1846 at high confidence. We consider high-resolution spectra of the planetary nebula Mo-17, and conclude that this object is also a member of the cluster. Our measured radial velocities allow us to conduct a detailed investigation of the internal kinematics of NGC 1846, the first time this has been done for an EMSTO system. The key result of this work is that the cluster exhibits a significant degree of systemic rotation, of a magnitude comparable to the mean velocity dispersion. Using an extensive suite of Monte Carlo models we demonstrate that, despite our relatively small sample size and the substantial fraction of unresolved binary stars in the cluster, the rotation signal we detect is very likely to be genuine. Our observations are in qualitative agreement with the predictions of simulations modeling the formation of multiple populations of stars in globular clusters, where a dynamically cold, rapidly rotating second generation is a common feature. NGC 1846 is less than one relaxation time old, so any dynamical signatures encoded during its formation ought to remain present.
\end{abstract}

Key words: globular clusters: individual (NGC 1846) - Magellanic Clouds - stars: kinematics and dynamics

Online-only material: color figures

\section{INTRODUCTION}

\subsection{Multiple Populations in Galactic Globular Clusters}

One of the outstanding problems in modern astrophysics concerns the formation of globular clusters. Long thought to constitute a simple, homogeneous class of object, each one consisting of stars of a uniform age and elemental composition, it is now recognized that these systems harbor multiple stellar populations displaying a wide variety of unexpected characteristics (see Gratton et al. 2012 for a review).

All Galactic globular clusters for which large samples of members have been studied spectroscopically at sufficiently high resolution and signal-to-noise ratio $(\mathrm{S} / \mathrm{N})$ are found to be comprised of stars exhibiting a characteristic chemical signature - a strong anti-correlation between the abundances of the light elements $\mathrm{O}-\mathrm{Na}$, as well as $\mathrm{C}-\mathrm{N}$ and $\mathrm{Mg}-\mathrm{Al}$ in many cases, even while remaining homogeneous in iron content (see, e.g., Carretta et al. 2009a, 2009b, 2009c). Since this pattern is observed in stars on the main sequence as well as in red giants, it must be primordial rather than being the result of nucleosynthesis and mixing within the observed giant stars (e.g., Gratton et al. 2004). The pattern is also seen in ancient globular clusters in nearby galaxies-for example, in the Large Magellanic Cloud (Hill et al. 2000; Johnson et al. 2006; Mucciarelli et al. 2009) and the Fornax (Letarte et al. 2006) and Sagittarius (e.g., Carretta et al. 2010a, 2010b) dwarfs-but not observed in old open clusters (with the recent exception of NGC 6791, Geisler et al. 2012) or for the vast majority of the Milky Way field halo (see, e.g., Martell \& Grebel 2010; Martell et al. 2011 and references therein), implying that it

\footnotetext{
* Based on observations obtained at the European Southern Observatory Very Large Telescope, Paranal, Chile, under programme 082.D-0387.
}

is a product specifically linked to globular cluster formation processes.

Beyond the anti-correlated light element abundance variations many globular clusters exhibit various additional levels of inhomogeneity, such as split main sequences or multiple subgiant branches on their color-magnitude diagrams (CMDs; e.g., Bedin et al. 2004; Piotto et al. 2007; Villanova et al. 2007; Milone et al. 2008), internal dispersions in the abundance of iron or other elements (e.g., Yong \& Grundahl 2008; Da Costa et al. 2009; Ferraro et al. 2009; Cohen et al. 2010; Mucciarelli et al. 2012), and/or subpopulations enhanced in helium (e.g., Norris 2004; Piotto et al. 2005; Dupree et al. 2011; Pasquini et al. 2011).

The overall picture is of a huge wealth of complexity that poses serious challenges for models of globular cluster formation and evolution. The pervasive light element anticorrelations require material processed at high temperatures via proton capture reactions. At $T \gtrsim 2 \times 10^{7} \mathrm{~K}$ the $\mathrm{CNO}$ and $\mathrm{NaNe}$ cycles serve to alter the carbon, nitrogen, oxygen and sodium abundances, while at somewhat higher temperatures the $\mathrm{MgAl}$ cycle also becomes active, leading to enhanced aluminum and reduced magnesium abundances. In the presently favored model, material processed in this way in a first generation of stars pollutes or forms a central accumulation of gas in a young globular cluster, and a second generation of stars bearing the characteristic light element signature is subsequently formed from this reservoir (see, e.g., D'Ercole et al. 2008; Conroy \& Spergel 2011). Note that because the iron content in most clusters is observed to be homogeneous, the gas should typically not have undergone supernova enrichment. Leading suggestions for the sites of the high-temperature processing are intermediatemass asymptotic giant branch (AGB) stars (e.g., Ventura et al. 2001) and fast-rotating massive stars (FRMS; e.g., Decressin 
et al. 2007). In both cases helium enhancement may also be introduced to the gas reservoir as a result of main-sequence hydrogen burning in the polluting stars.

A generic prediction of this model is that the second generation of stars in a globular cluster ought to be more centrally concentrated than the first generation, and this seems to have been observed in some cases (Lardo et al. 2011). However, the model also suffers from a number of difficulties, chief among which is that the second generation stars in globular clusters require significantly more gas for formation than can have been available based on the presently observed numbers of first generation members. Ideas invoked to circumvent this issue include the accretion of large amounts of pristine interstellar material (e.g., Conroy \& Spergel 2011), a first generation 10-100 times more massive than present-day globular clusters (e.g., D'Ercole et al. 2008; Bekki 2011), a first generation with a top-heavy initial mass function (IMF; e.g., D’Antona \& Caloi 2004; Bekki \& Norris 2006), or a variation in which globular clusters are formed as part of initially larger systems such as low-mass dwarfs (e.g., Bekki et al. 2007). Beyond this difficulty, it is also unclear how clusters with internal dispersions in iron-peak elements or that include populations with very high helium abundances $(Y \approx 0.4)$ fit into the model.

The unavoidable conclusion from the scenario outlined above is that the formation of individual globular clusters must have spanned a period of tens to hundreds of Myr, depending on the nature of the stars responsible for the high-temperature processing. With presently available facilities, however, we are unable to directly resolve age differences of this magnitude given that Galactic globular clusters are typically $\sim 12$ Gyr old. In addition, since the timescale for dynamical relaxation in the majority of Galactic globular clusters is much shorter than the cluster age (see Harris 1996) any detailed information imprinted on the internal kinematics of these systems as a result of the formation process will have long since been seriously diluted or possibly erased altogether.

\subsection{The Role of Peculiar Magellanic Cloud Clusters}

Star clusters in the Large and Small Magellanic Clouds (LMC and SMC) offer an important new piece of this puzzle. These two galaxies possess extensive systems of clusters spanning the full age range $\sim 10^{6}-10^{10}$ years; many of the members of these systems are comparable in mass to present-day Galactic globular clusters lying at or below the peak of the luminosity function (see, e.g., Mackey \& Gilmore 2003a, 2003b). Using images taken with the Advanced Camera for Surveys (ACS) on board the Hubble Space Telescope (HST), we recently demonstrated that several rich intermediate-age clusters $(\tau \sim 1.5-2$ Gyr) in the LMC display markedly unusual CMDs (Mackey \& Broby Nielsen 2007; Mackey et al. 2008a). More specifically, we found that while the CMDs for NGC 1783, 1806 and 1846 have very narrow main sequences and red giant branches (RGBs), the main-sequence turnoffs (MSTOs) for these clusters are much broader than can be explained by the photometric uncertainties (see Figure 1). After considering various possibilities for the origin of these extended main-sequence turnoffs (EMSTOs), such as confusion due to unresolved binary stars or field contamination, we concluded that the simplest viable interpretation of our observations is that each of the three clusters is comprised of two or more stellar populations with very similar iron abundance but spanning an age interval of $\approx 300 \mathrm{Myr}$.

Subsequent work has reinforced this interpretation and revealed that an EMSTO is apparently not an unusual feature for intermediate-age Magellanic Cloud clusters. Photometric analysis of HST imaging by Milone et al. (2009) and Goudfrooij et al. $(2009,2011 \mathrm{~b})$ demonstrated that, of the 16 intermediateage LMC clusters with suitable data, 11 possess EMSTOs (see also Mackey et al. 2009). In addition, Glatt et al. (2008) discovered that the intermediate-age SMC cluster NGC 419 possesses an EMSTO. Each of these studies concluded that an internal age spread was the most likely explanation for these features, with the full sample of EMSTO clusters encompassing the range $\sim 150-500$ Myr for this spread.

Additional support has come from several directions. Girardi et al. (2009) noted the presence of a dual red clump in NGC 419 and in a number of the rich LMC EMSTO clusters, while Rubele et al. (2010, 2011) used the complete observed CMDs for NGC 419 and NGC 1751 to reconstruct their star-formation histories-finding that these may have spanned an incredible $\sim 700 \mathrm{Myr}$ (out of a mean age of $\sim 1.5 \mathrm{Gyr}$ ) in NGC 419 , and $\sim 460 \mathrm{Myr}$ in NGC 1751. Rubele et al. $(2010,2011)$ noted that in their best-fitting models the dual red clumps in both NGC 419 and NGC 1751 arise as a direct result of the age dispersions in these systems. Bastian \& de Mink (2009) posited that rather than reflecting an internal age spread, a cluster EMSTO might instead arise if a wide range in stellar rotation is present at the MSTO. However, models by Girardi et al. (2011) demonstrated that the effects of stellar rotation fail to reproduce the EMSTO morphology; in addition, as emphasized by Rubele et al. (2010, 2011), this scenario does not seem able to account for the dual red clumps observed in NGC 419, NGC 1751, and some other EMSTO clusters. Girardi et al. (2011) also tested the effects of star-to-star variations in the degree of convective overshooting on a cluster CMD, but found that this too failed to accurately reproduce the characteristic EMSTO shape.

The global properties of EMSTO clusters provide some clues as to how these systems may arise. Conroy \& Spergel (2011) made the simple observation that it is only the intermediateage clusters with present-day masses greater than $\approx 10^{4} M_{\odot}$ that exhibit EMSTOs. Keller et al. (2011) further observed that the known EMSTO systems are, without exception, the most diffuse, spatially extended clusters for their age. In the framework of Elson et al. (1987) and Mackey \& Gilmore (2003a, 2003b), Magellanic Cloud clusters exhibit an increasing spread in size (defined by either the core or half-light radius) with age; Keller et al. (2011) showed that at intermediate ages the EMSTO clusters all fall toward the upper envelope of this distribution, while clusters without an EMSTO appear systematically more compact. Mackey et al. (2008b) used detailed $N$-body models to explore the origin of the increasing spread in Magellanic Cloud cluster sizes with age. They found that if very young massive clusters are formed as compact dense systems, as is seen to be the case in both the LMC and SMC, the only viable way for them to evolve along the upper envelope of the observed age-size distribution on a $\lesssim 1$ Gyr timescale is due to massloss from stellar evolution if they were initially highly mass segregated - that is, with the highest mass stars preferentially located toward the cluster centers. ${ }^{3}$ This in turn implies that, in addition to being the most massive intermediate-age clusters

\footnotetext{
3 Mackey et al. (2008b) found that a retained population of stellar mass black holes can also cause a cluster to move toward the upper envelope of the age-size distribution, but on longer timescales than 1-2 Gyr. Note also that Elson et al. (1989) found that a flat, or top-heavy IMF can lead to cluster expansion along the upper envelope of the age-size distribution; however, such clusters rapidly become unbound (after just a few $\times 10^{7} \mathrm{yr}$ ). In addition, young Magellanic Cloud clusters are seen to have quite normal IMFs (e.g., Kroupa 2001; de Grijs et al. 2002).
} 

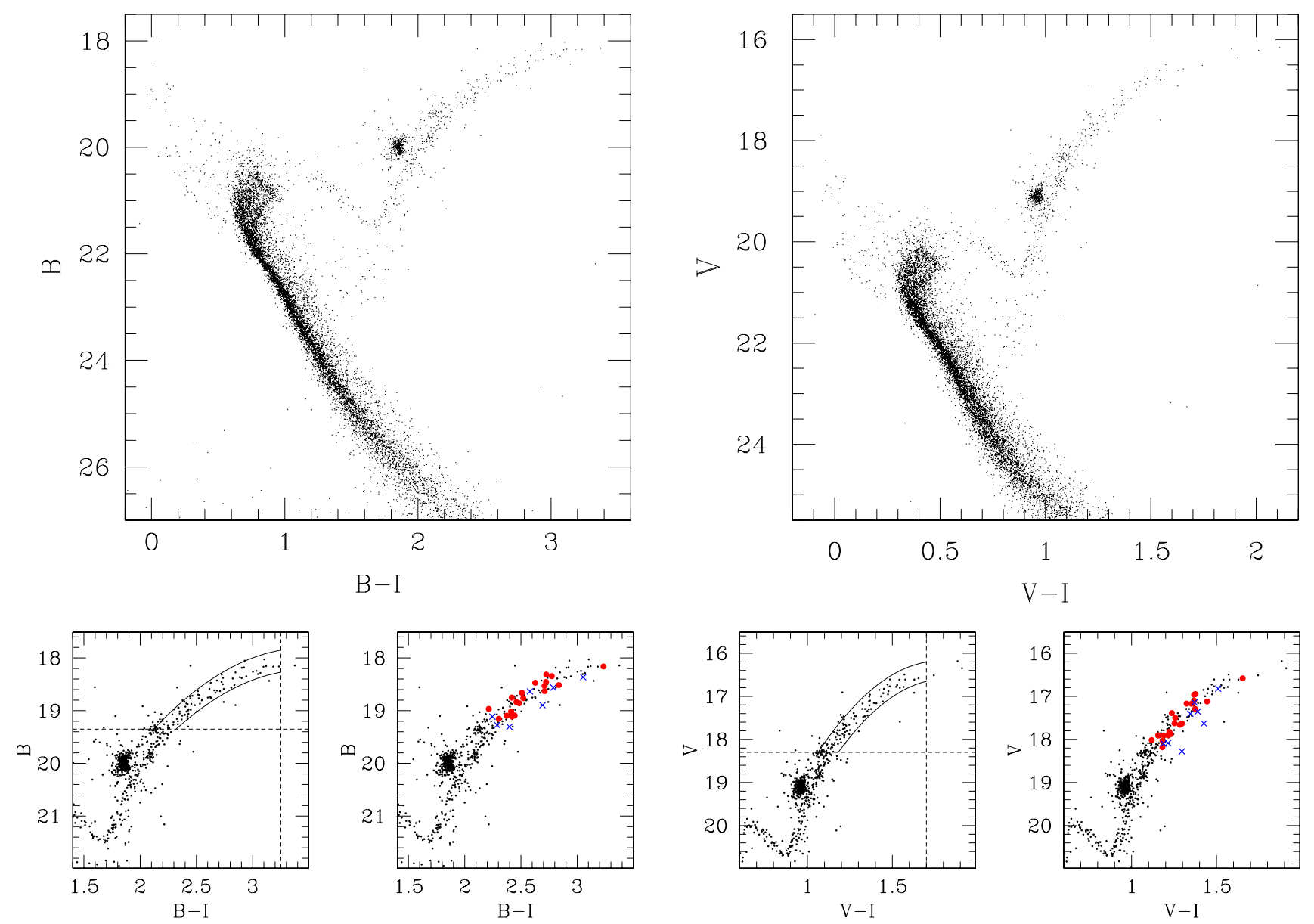

Figure 1. HST/ACS color-magnitude diagrams for NGC 1846, in the $(B-I, B)$ and $(V-I, V)$ planes. In the upper two panels, the peculiar extended main-sequence turnoff morphology is clearly evident. The lower four panels show the regions on the red giant branch for our FLAMES target selection, bounded by dashed and solid lines, along with the objects we observed. Those targets marked with red dots are radial velocity members of the cluster (see Section 3.1); those marked with blue crosses are not.

(A color version of this figure is available in the online journal.)

in the Magellanic Clouds, EMSTO systems were also probably the most strongly mass segregated clusters at early times. There is clearly a link between cluster mass and structure, and the presence of an EMSTO.

There are two additional observations of relevance. If a cut is made on the CMD across a cluster's EMSTO in a direction perpendicular to the locus of the upper main sequence, then groups of "younger" and "older" MSTO stars may be defined. These groups have distinct properties. First, as noted by Milone et al. (2009), the younger population consists of at least as many stars as the older population-in fact the ratio is typically more like 2:1 in the richest clusters. Second, as described in detail by Goudfrooij et al. (2011a), the younger population is often more centrally concentrated than the older population. The strongest difference between the two concentrations is seen in the clusters possessing the largest estimated initial escape velocities (i.e., $v_{\text {esc }} \gtrsim 15 \mathrm{~km} \mathrm{~s}^{-1}$ at an age of $\sim 10 \mathrm{Myr}$ ); systems in which $v_{\text {esc }} \lesssim 10 \mathrm{~km} \mathrm{~s}^{-1}$ do not show this difference in central concentrations, or are not seen to possess an EMSTO at all (note that the limiting velocity here is comparable to the velocities of winds from FRMS or AGB stars). This picture is fully consistent with the idea discussed above that EMSTO clusters were both the most massive and the most strongly mass segregated clusters at the time when they were formed.

As speculated by a number of authors (Conroy \& Spergel 2011; Goudfrooij et al. 2011a; Keller et al. 2011), the properties of EMSTO systems suggest a formation process remarkably similar to that inferred for the multi-population Galactic globular clusters-specifically, that prolonged star formation has occurred at the centers of these objects because their masses and initial structures allowed the retention or accumulation of a suitable reservoir of gas at the bottom of the cluster potential well. The observed internal age spreads of several hundred Myr in EMSTO clusters, and the apparently minimal dispersions in iron abundance inferred from their narrow RGB sequences are both consistent with the scenario invoked for Galactic globular clusters in which much of this reservoir comes from the slow winds of a first generation of AGB stars. Furthermore, the number ratio of younger to older groups of stars in EMSTO clusters is comparable to that seen for the two generations in Galactic globular clusters. Note that it has not been clearly assessed how early ideas for the formation of EMSTO systems, such as the merging of two bound clusters (e.g., Mackey \& Broby Nielsen 2007) or the merger of a cluster and a giant molecular cloud (Bekki \& Mackey 2009), fit with the observed properties of EMSTO systems. However, if the star formation has indeed progressed unbroken over several hundred Myr as suggested by Rubele et al. (2010, 2011) or Goudfrooij et al. (2011b), these scenarios are likely disfavored.

If, as hypothesized, EMSTO clusters and Galactic globular clusters share a common formation process, a key prediction is that EMSTO clusters ought to harbor similar star-to-star 
variations in the abundances of light elements as seen in the Galactic systems. A complicating factor is the comparatively high overall metallicity of the EMSTO clusters $([\mathrm{M} / \mathrm{H}] \approx$ -0.4 ), along with the possibility that their central gas reservoirs may have been augmented by an unknown amount of accreted pristine material (e.g., Conroy \& Spergel 2011)—so the scale of these variations is difficult to predict. Very few light element abundance measurements exist for EMSTO systems. The most extensive study is that by Mucciarelli et al. (2008) who targeted between 5 and 11 stars in each of four intermediate-age LMC clusters, of which only one (NGC 1783) unambiguously features an EMSTO. For all four systems, Mucciarelli et al. assert that the star-to-star scatter in each of the $\sim 20$ elemental abundances that they measure is negligible; in particular, there are no O-Na or $\mathrm{Mg}-\mathrm{Al}$ anti-correlations evident. However, the interpretation of these measurements is somewhat controversial-both Conroy (2012) and Goudfrooij et al. (2011a) note that the star-to-star scatter in the listed abundances of sodium is larger than the observational uncertainties at a statistically significant level (up to $\sim 4 \sigma$ ) for some clusters. Even so, the sole EMSTO cluster in the study, NGC 1783, does not seem to exhibit elemental abundance patterns which are strikingly distinct from those of the other three systems.

\subsection{This Work}

Additional high-resolution spectral data, for a larger sample of stars in a larger ensemble of EMSTO clusters, are clearly required to assess the viability of the link between these systems and the multi-population Galactic globular clusters. Such data possess an extra benefit beyond exploring elemental abundance patterns. Because EMSTO clusters are diffuse, low-density stellar systems, their two-body relaxation times are typically comparable to or longer than their ages (see, e.g., Goudfrooij et al. 2011a). Thus, unlike for Galactic globular clusters, the internal dynamics of EMSTO systems should still reflect the conditions present early on in their lives — so that any signature imparted onto the cluster kinematics by the formation process should be both detectable and straightforward to interpret.

This paper is the first in a series devoted to a detailed study of medium and high-resolution spectra for 21 giant stars and one planetary nebula in the most massive known EMSTO cluster, and one of the best studied photometrically-NGC 1846 in the LMC. Stellar populations in this cluster span the age range $\approx 1.6-1.9$ Gyr, and its iron abundance is $[\mathrm{Fe} / \mathrm{H}] \approx-0.4$ (e.g., Mackey \& Broby Nielsen 2007; Mackey et al. 2008a). The aim of our study of NGC 1846 is twofold: first, to characterize the internal dynamics of the cluster and search for any signatures of its formation that might be present, and second, to place constraints on any star-to-star elemental abundance variations, especially for light elements. Here, we present a detailed description of our target selection and data analysis (Sections 2 and 3), and focus on the cluster kinematics (Sections 4 and 5). Subsequent work will cover the elemental abundance analysis.

\section{OBSERVATIONS AND DATA REDUCTION}

\subsection{Data Acquisition}

We obtained spectra of stars in the vicinity of NGC 1846 using the FLAMES instrument at the ESO Very Large Telescope (VLT) on Cerro Paranal, Chile, under programme 082.D-0387 (PI: Mackey). FLAMES (Pasquini et al. 2002) is a fiber-fed multi-object spectrograph mounted at the Naysmith A platform of the $8.2 \mathrm{~m}$ Unit Telescope 2 (Kueyen). We employed the
Table 1

Nominal Wavelength Coverage and Spectral Resolution for Our Four GIRAFFE Setups

\begin{tabular}{lcccr}
\hline \hline $\begin{array}{l}\text { Configuration } \\
\text { Name }\end{array}$ & $\begin{array}{c}\lambda_{\text {start }} \\
(\AA)\end{array}$ & $\begin{array}{c}\lambda_{\text {end }} \\
(\AA)\end{array}$ & $\begin{array}{l}\Delta \lambda \\
(\AA)\end{array}$ & $R$ \\
\hline HR11 & 5597 & 5840 & 243 & 24200 \\
HR13 & 6120 & 6405 & 285 & 22500 \\
HR14B & 6383 & 6626 & 243 & 28800 \\
LR02 & 3964 & 4567 & 603 & 6000 \\
\hline
\end{tabular}

MEDUSA-GIRAFFE mode, allowing up to 132 stars to be targeted across the $25^{\prime}$ diameter field of view in a single pointing.

Our target selection is discussed in detail in Section 2.2. We used just a single FLAMES fiber configuration but observed at three high-resolution GIRAFFE settings (HR11, HR13, and HR14B) and one low-resolution setting (LR02). The nominal wavelength coverage and spectral resolution for each of these setups is listed in Table 1. This paper and the next in the series (Paper II) are dedicated to analysis of the observations obtained at the three HR settings; results from the blue LR setup will be reported in a separate future work.

We obtained our data in visitor mode on the three nights 2008 November 29-December 1. Conditions were clear and stable, with seeing typically in the range $0.5-1{ }^{\prime \prime} .0$. On each night we observed a given HR setting at the beginning and end of the night, and reserved an hour either side of NGC 1846 crossing the meridian for observing the blue LR setup with minimal differential atmospheric refraction. For all four gratings we obtained $6 \times 55 \mathrm{~min}$ exposures. Due to the faintness of our targets we switched the simultaneous calibration lamps off, instead bracketing each long science exposure with short calibration lamp exposures to ensure we could achieve an accurate wavelength solution.

\subsection{Target Selection and Photometry}

Targets for our FLAMES observations were drawn from the archival HST / ACS imaging of NGC 1846 described in the introduction (Mackey \& Broby Nielsen 2007; Goudfrooij et al. 2009) for the crowded central regions, and the Magellanic Clouds Photometric Survey (MCPS) catalog of the LMC (Zaritsky et al. 2004) for the surrounding field.

For work such as this, one would ideally directly target stars spread across the cluster EMSTO so that elemental abundances and dynamical properties can be correlated directly against position on the CMD. Unfortunately, however, at $V \approx 20.5$, obtaining sufficient $\mathrm{S} / \mathrm{N}$ for a high-precision abundance analysis of NGC 1846 MSTO stars is beyond the capabilities of presently available high-resolution spectrographs, even on the largest telescopes. Instead, we are forced to target the brightest giant stars in the cluster. There is, however, no major disadvantage in doing so-Milone et al. (2009) demonstrated that the relative numbers of "younger" and "older" stars across the EMSTO are not too dissimilar (a ratio of $\approx 2: 1$ ). This means that, providing that a sufficiently large ensemble of giant stars is observed, the full spread in population parameters (age and, if they exist, elemental abundance variations) ought to be well sampled.

At the time we were preparing our VLT observations, HST imaging of NGC 1846 was available from only two separate programs: 9891 (PI: Gilmore) and 10595 (PI: Goudfrooij), both of which utilized the ACS Wide Field Channel (WFC). The former is a "snapshot" program, where the imaging consists of just two exposures-300 s in the F555W filter and $200 \mathrm{~s}$ 
in the F814W filter. The second program comprises a more extensive set of observations - three exposures in each of the F435W, F555W, and F814W filters, where two of the exposures were long (340 s each) and one short $(90 \mathrm{~s}, 40 \mathrm{~s}$, and $8 \mathrm{~s}$ in the three filters, respectively). The two programs were observed with differing orientations, meaning that their footprints only partially overlap on the sky. In the interests of covering as much of the area around NGC 1846 as possible with highquality ACS imaging and photometry, and hence maximizing the number of objects in our input catalog for FLAMES target selection, we used the MULTIDRIZZLE software (Koekemoer et al. 2003) to combine the complete set of F555W observations into a "master" reference image. This image increases the sky coverage near NGC 1846 by $~ 30 \%$ over what would have been available using only one of the $H S T$ programs.

We next used the DOLPHOT software (Dolphin 2000), and in particular its ACS module, to photometer all the available images. Details of this procedure may be found in Mackey \& Broby Nielsen (2007). Briefly, DOLPHOT performs point-spread function (PSF) fitting photometry using model PSFs especially tailored for the ACS camera. It works on images for which basic reduction steps have been applied (bias and dark current subtraction, and flat-field division) but which have not been distortion corrected (drizzled). The software can photometer multiple images in multiple filters simultaneously, matching detections across images and deriving coordinates relative to an input reference frame-in our case the master drizzled F555W image. Output photometry is on the calibrated VEGAMAG scale of Sirianni et al. (2005) and has been corrected for charge-transfer efficiency degradation. Where possible, transformations are also made into the standard Johnson-Cousins system. To obtain a clean list of stellar detections with high-quality photometry we filtered our DOLPHOT measurements using the classification, sharpness, and crowding parameters (see Mackey \& Broby Nielsen 2007; Mackey et al. 2008a).

CMDs, in both the $(B-I, B)$ and $(V-I, V)$ planes are shown in Figure 1. NGC 1846 is set against a relatively dense background of LMC field stars, so care was necessary in selecting our input catalog for FLAMES. We used CMDs for stars within $30^{\prime \prime}$ of the cluster center to filter out most of the field contamination, allowing us to define regions encompassing the upper RGB and AGB on both color-magnitude planes. The color and luminosity of a star on the RGB is sensitive to elemental abundances, especially that of iron, as well as to age. Given that the primary aim of our VLT program was to test for possible star-to-star abundance variations, and that we also strongly suspect the presence of an internal age spread in NGC 1846, we were particularly mindful not to define our target regions too restrictively. The red side of the NGC 1846 RGB is sharply defined on both CMD planes; however, to the blue there is potential for significant overlap between the RGB and AGB sequences. Hence, we set the blue side of our target region rather conservatively, with the result that there are likely a few AGB stars in our FLAMES sample. As long as these are early-AGB objects (i.e., which have not yet undergone third dredge-up) the composition of the atmospheres of these stars should not have been altered substantially through the addition of newly-processed material. This issue is not relevant to the cluster kinematics, but is discussed in more detail in Paper II.

We set an absolute red limit to our target region of $V-I=1.7$ in order to exclude very cool giants for which it is more difficult to do a reliable abundance analysis. Note that this also excluded the most luminous (and evolved) AGB stars in the cluster. At the faint end we set a limit of $V=18.3$, as spectra for stars fainter than this would not have sufficiently high $\mathrm{S} / \mathrm{N}$. On the $(B-I, B)$ plane, the corresponding limits were $B-I=3.25$ and $B=19.35$.

Having defined our target regions we selected as our cluster input catalog all stars across the full ACS field of view that lay within these regions on both color-magnitude planes. As noted above, nearly a third of the field is not covered by F435W imaging - we filtered objects in this area using only the $(V-I, V)$ plane. The very outer parts of the ACS field were only sparsely populated with suitable stars, so we supplemented the input catalog in this region with stars lying up to $\sim 0.1 \mathrm{mag}$ to the red of our target region on the CMD. We also added to the catalog a nearby planetary nebula likely belonging to NGC 1846 , Mo-17 (see Section 3.2). We searched for and removed from the catalog any stars with neighbors within a radius of $2^{\prime \prime}$ that were sufficiently bright as to be likely to interfere with the spectrum of the target.

We used ESO's Fibre Positioner Observation Support Software (FPOSS) to determine the optimal MEDUSA configuration for our FLAMES observations. A few trial runs using our ACS input catalog demonstrated that we would be able to observe, at most, $\sim 30$ stars within $\approx 2^{\prime}$ of the center of NGC 1846 - the limiting factor being the minimum fiber-tofiber separation of $11^{\prime \prime}$. Even allowing for $\sim 15$ sky fibers and $\sim 5$ fibers allocated to hot blue stars to allow correction of telluric absorption (see below), this left roughly 80 fibers unused. Rather than waste these we decided to allocate them to bright field RGB stars spread evenly over the non-cluster regions of the $25^{\prime}$ diameter FLAMES field of view.

We selected these objects from the MCPS catalog of the LMC (see Zaritsky et al. 2004). This catalog provides precise astrometry and $U B V I$ photometry of stars in the central 64 $\mathrm{deg}^{2}$ of the LMC, and, for some subset of successfully crossmatched stars, $J H K_{s}$ photometry from the Two Micron All Sky Survey (2MASS) catalog (Skrutskie et al. 2006). There were a sufficiently large number of MCPS stars with 2MASS matches across our field of view that we were able to draw targets exclusively from this subset. The 2MASS measurements were particularly useful for distinguishing between field RGB and AGB stars, as shown in Figure 2 (left panel). We selected RGB stars brighter than $V \approx 17$ for our MCPS input catalog (Figure 2, right panel).

Available literature measurements suggested that the NGC 1846 radial velocity of $V_{\text {sys }} \approx 240 \mathrm{~km} \mathrm{~s}^{-1}$ (Olszewski et al. 1991; Grocholski et al. 2006) would bring the forbidden [O I] line at $6300.3 \AA$ into close proximity with telluric absorption features due to atmospheric $\mathrm{O}_{2}$. We planned to derive our oxygen abundances using primarily this line (see Paper II); in order to be able to account for the telluric absorption we required spectra of hot, preferably fast-rotating stars at high $\mathrm{S} / \mathrm{N}$. To this end, we selected a number of target stars from the MCPS catalog that appeared to be bright members of the young blue LMC field main sequence (see Figure 2).

To aid in the subtraction of atmospheric emission lines from our science spectra, we also decided to allocate $\approx 15$ fibers to blank sky. We placed these such that for a given sky fiber there were no objects in the MCPS catalog lying within $5^{\prime \prime}$. Ideally these sky positions would be local to our highest priority targets (i.e., NGC 1846 members); however, the very tightly packed nature of the fiber configuration in the middle of the FLAMES field meant that it was impossible to allocate any other fibers within $\sim 5^{\prime}$ of the cluster center. This ultimately led to some 

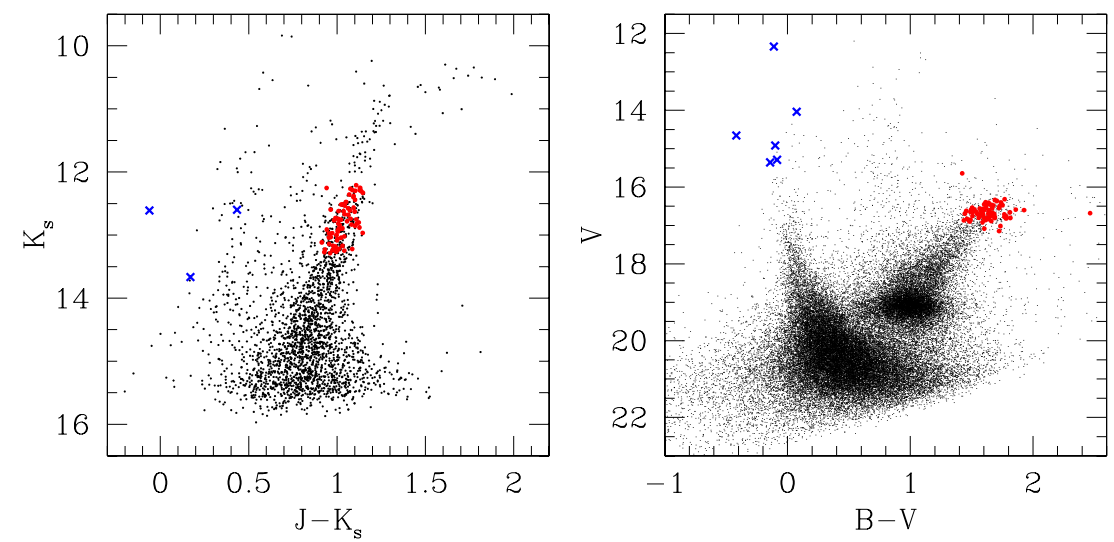

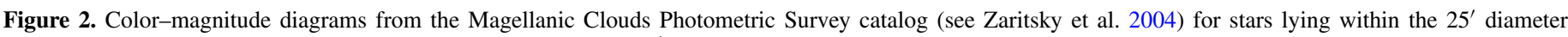

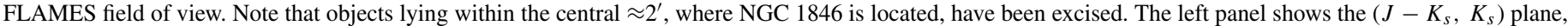

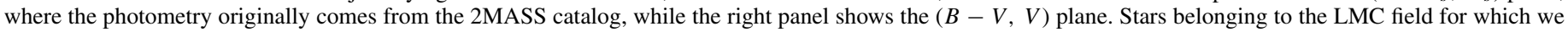

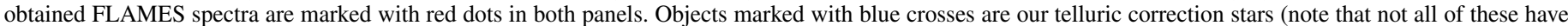
infrared photometry).

(A color version of this figure is available in the online journal.)

problems achieving a high-quality sky subtraction for many of our targets; however, it was not difficult to accommodate these issues in our analysis (Section 2.3).

Finally, we merged our ACS and MCPS catalogs for input to FPOSS. The critical step was to transform the ACS coordinates onto the MCPS (FK5) astrometric frame. Although the relative astrometry of targets measured from the ACS imaging is extremely precise (of the order of milliarcseconds), the HST FITS header information from which the absolute astrometry is derived can be in error by up to several arcseconds (e.g., Anderson et al. 2008)—more than enough for the MEDUSA fibers to miss these targets entirely. We cross-matched $\sim 100$ stars across the ACS master reference image with stars in the full MCPS LMC catalog, excluding the crowded central cluster region, and used these to derive a suitable coordinate transformation onto the MCPS frame.

Having successfully merged our two lists of potential targets, we used FPOSS to determine the optimal fiber configuration. In doing so, we assigned priorities to the various classes of target-for example, ACS stars received higher priority than MCPS stars and sky positions. The ACS stars themselves were graded in priority by luminosity (the brighter the better), distance from the center of the cluster (the smaller the better), and, weakly, by color on the RGB (the redder the better).

Our final configuration targeted 30 ACS stars (11 within 50" of the cluster center), the planetary nebula Mo-17, 79 MCPS field RGB stars, 6 MCPS stars for correcting telluric absorption, and 16 blank sky positions. We observed our blue LR setting using FLAMES fiber positioner plate 1 and the three HR settings using plate 2 . On both plates, spectra from two fibers (both targeting MCPS stars) fell off the edge of the CCDs and were lost. Additionally, two of the plate 2 fibers were broken leading to the loss of another MCPS star and one ACS target. Hence, the HR observations presented here resulted in spectra for 76 MCPS stars and 29 ACS stars, as well as the other targets and sky positions listed above.

\subsection{Data Reduction}

We used the ESO public GIRAFFE pipeline recipes v2.8.1, operating under the graphical front-end software GASGANO, to perform a basic reduction of all our science frames-that is, bias subtraction, fiber localization, optimal extraction of spectra, division by a normalized flat-field image (i.e., including correction for fiber-to-fiber transmission differences), an initial wavelength calibration, and rebinning to a uniform linearized dispersion scale $(0.05 \AA$ per pixel for the spectra considered here). For each of the four instrumental setups, the science frames included the six on-target exposures as well as the short bracketing exposures with the calibration lamps on. For science frames belonging to a given setup, the initial wavelength calibration was derived from an arc-lamp exposure taken at the beginning of the night on which the science frames were taken. After the basic data reduction had been completed, we used the measured positions of emission lines in the bracketing calibration frames to check for small residual wavelength drifts in each of the on-target exposures individually.

Next, we intended to perform a sky subtraction on each individual spectrum of an object, along with a correction for telluric absorption, before combining these spectra into a final product. We note that sky subtraction is only marginally important for the HR11 setup, which covers a handful of weak emission lines; however the HR13 and HR14B setups both cover numerous bright emission lines. Similarly, telluric absorption is negligible $(\lesssim 3 \%)$ for the HR11 and HR14B setups, but is noticeable for the HR13 setup over the range 6275-6330 where there are many lines of $\sim 5 \%-15 \%$ absorption due to atmospheric $\mathrm{O}_{2}$.

Despite testing a variety of techniques we were unable to obtain a high-quality sky subtraction across the full wavelength coverage of either the HR13 or HR14B setups, especially for the likely members of NGC 1846 . Using the spectrum from the nearest sky fiber to a target did not work, nor did subtracting a combined spectrum derived from all 16 sky fibers in a given exposure. Using the IRAF task SKYTWEAK to vary the scaling of the sky spectra and apply small wavelength shifts improved the results marginally, but not to a satisfactory level. The origin of the problem is unclear, but it may well be linked to our inability to place sky fibers very locally to the NGC 1846 targets, as described in Section 2.2.

To work around this issue we used the IRAF task SCOMBINE to merge all 96 sky spectra for a given setup (16 sky fibers from each of 6 exposures) into a high-S/N "master" spectrum, and used this to generate a mask specifying all of the narrow 

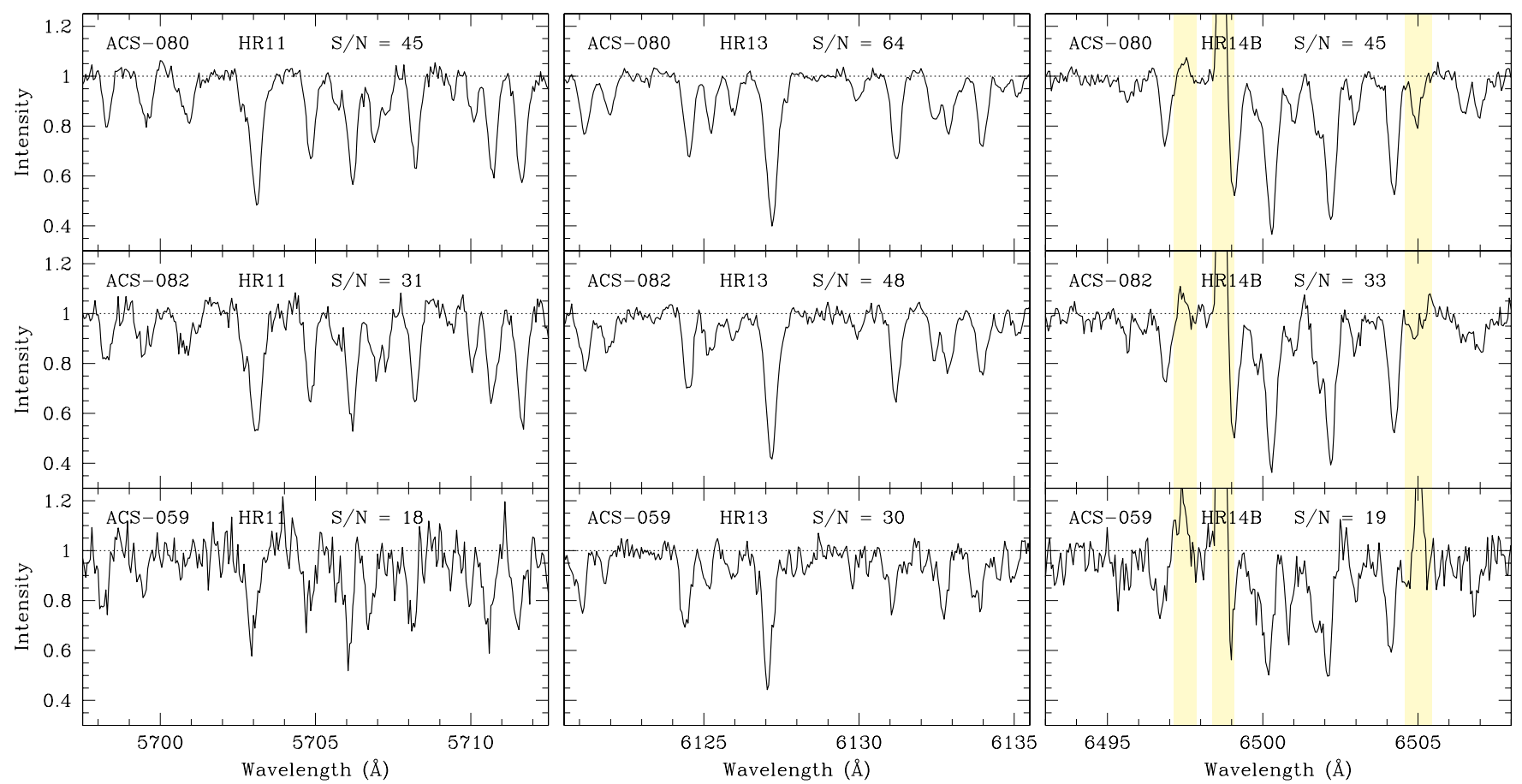

Figure 3. Representative spectral segments for three of our confirmed NGC 1846 members, as labeled. Each segment spans $\approx 15 \AA$. The left column contains a region from the HR11 coverage, the central column from the HR13 coverage, and the right column from the HR14B coverage. The spectra have been normalized following our DAOSPEC analysis (see Section 2.4) and corrected to the heliocentric frame; however, no correction has been made for the individual radial velocities. Regions masked in our analysis due to the presence of sky lines are shaded yellow (note that in this example such regions appear in the HR14B spectra only). The top row of the plot corresponds to one of the brightest targets in the NGC 1846 sample, and the spectra are of commensurately high S/N. Similarly, the bottom row shows spectra for one of the faintest targets, and these are of correspondingly low S/N. The middle row contains data of intermediate $\mathrm{S} / \mathrm{N}$; two-thirds of our sample of confirmed NGC 1846 members have spectra of comparable or higher S/N. Note that even in the lowest S/N spectra, most absorption lines are still clearly visible.

(A color version of this figure is available in the online journal.)

wavelength intervals affected by sky lines. Across the wavelength range of the HR13 setup there were 33 such intervals in the mask, covering $16.7 \%$ of any given spectrum, while for HR14B there were 25 sky-line intervals excising $11.1 \%$ of the coverage and for HR11 there were 8 intervals masking $3.3 \%$. When detecting and measuring stellar absorption features for our kinematic and chemical abundance analysis, we simply ignored any lines lying within one resolution element $(\sim 0.3 \AA)$ of a masked sky region. As described in Paper II, this procedure did not reject any lines critical for examining the abundance of a given element, but did ensure that any lines impacted by sky emission did not adversely influence our measurements.

We encountered a similar problem when attempting to correct for telluric absorption in the HR13 setup. Four of our six bright blue targets turned out to be suitable stars for this type of correction; however, despite trying a similar variety of techniques as for the sky subtraction, we could not consistently achieve a high-quality correction across the full affected wavelength range. Ultimately, we adopted an identical approach to our sky-line solution-we normalized then combined the 24 telluric star exposures into a high-S/N master spectrum and used this to generate a mask specifying the intervals affected by atmospheric absorption lines. There were 13 such intervals covering $10.1 \%$ of the HR13 wavelength range; we note some overlap between the telluric and sky-line masks. Again, during our kinematic and chemical abundance analysis we simply ignored any stellar lines lying within one resolution element of a masked telluric-line region. As described in more detail in Paper II, the only critical stellar line rejected by this procedure was the [O I] line at $6300.3 \AA$, which, for NGC 1846 targets, is redshifted onto a telluric feature near $6305.5 \AA$. In order to use this line, we applied our master spectrum and the IRAF task TELLURIC to obtain a high-quality correction to only the $6305.5 \AA$ feature, and then used these specially tailored spectra when deriving oxygen abundances.

Finally, we corrected all on-target spectra to the heliocentric frame using the IRAF tasks RVCORRECT and DOPCOR. We then median-combined the six exposures of a given target in a given setup using the IRAF task SCOMBINE. Best results (including cosmic ray removal) were achieved by scaling the input spectra to a common flux level and then, when combining them, weighting each by its median flux value and applying a sigmaclipping rejection algorithm. Wavelength regions covered by the sky- and telluric-line masks were excluded when computing the scaling and weighting factors.

Example spectra for three NGC 1846 stars spanning nearly the full range in brightness of our sample of confirmed members (see Section 3.1) are shown in Figure 3. We estimated the continuum $\mathrm{S} / \mathrm{N}$ for these objects with the IRAF task SPLOT. The setting with the highest $\mathrm{S} / \mathrm{N}$ per pixel is HR13; spectra for the HR11 and HR14B settings have S/N levels consistently 35\%-40\% lower for the same exposure duration. Partly this is due to the slightly higher resolution of these two settings (the HR11 and HR14B settings span wavelength ranges $\approx 15 \%$ shorter than that for HR13 - see Table 1) and partly it is due to their somewhat lower overall efficiency. The HR13 spectra span S/N 30-70 per pixel, while the HR11 and HR14B spectra have $\mathrm{S} / \mathrm{N}$ in the range $\sim 18-50$ per pixel. For reference, the HR13 setting has $\approx 5.6$ pixels per resolution element at the midpoint of its coverage, whereas HR11 has $\approx 4.7$ and HR14B $\approx 4.5$. Overall, two-thirds of our confirmed NGC 1846 stars have spectra with 
$\mathrm{S} / \mathrm{N}$ comparable to, or greater than, the middle star plotted in Figure 3.

\subsection{Line Identification and Measurement}

We next applied the DAOSPEC software package to each of our $3 \times(29+76)=315$ GIRAFFE HR spectra. DAOSPEC is an automated tool, optimized for spectra with $R \gtrsim 15,000$, for identifying and measuring absorption lines. Full details can be found in Stetson \& Pancino (2008), but briefly, it works by iteratively finding lines in a given spectrum, fitting these with Gaussian profiles of fixed FWHM and subtracting them, and then using the residual spectrum to refine the continuum normalization and improve the line centroids and strengths. Once this process has converged, the measured lines are crosscorrelated against a user-supplied line list to provide an estimate of the radial velocity of the target along with an identification for any lines that are successfully matched. The accuracy of DAOSPEC measurements, especially for equivalent widths, has been tested and verified on GIRAFFE HR data (e.g., Pompéia et al. 2008; Letarte et al. 2010).

Full details of our equivalent width measurements are provided in Paper II; here we are mainly interested in the radial velocities supplied by DAOSPEC. We assembled an input line list and atomic data for 18 neutral and 12 singly ionized species (25 elements in total) over the wavelength interval 5550-6650 using the VALD atomic line database (Kupka et al. 1999). We included only the "strongest" lines for each species, where strength was approximately parameterized by the difference between the oscillator strength $\log (g f)$ and the excitation potential $\chi$ (i.e., by the sum $\log (g f)-\chi$ ). For each species an empirical minimum limit for this value was defined by manually examining lines in our highest $\mathrm{S} / \mathrm{N}$ spectra and identifying those with the smallest equivalent widths that could be reliably identified and measured (typically $\mathrm{EW} \approx 15 \mathrm{~m} \AA$ ). Our final line list contained a total of 1445 lines over the $1100 \AA$ wavelength interval. Before input to DAOSPEC we applied our sky and telluric masks to cull affected lines from the list.

When measuring the radial velocity of a target, DAOSPEC uses a sigma-clipping rejection algorithm to eliminate identified lines with discrepant velocities. In order to determine precise radial velocities for our stars we performed an initial run on all spectra with stringent $2 \sigma$ rejection. This was important because at this stage we had not checked our line list for features that would be blended at the GIRAFFE HR resolutions. As described in Paper II, for the equivalent width measurements we subsequently performed a more relaxed $3.5 \sigma$ rejection run and then determined the most appropriate lines for elemental abundance analysis by careful visual inspection of the spectra. However important additional guidance was provided by the identification of those lines initially rejected in the stringent $2 \sigma$ DAOSPEC run.

For each of our targets we ultimately obtained three independent radial velocity estimates from DAOSPEC, one for each HR setting. Typically, $\approx 130$ lines were identified in each HR11 spectrum, $\approx 100$ lines in each HR13 spectrum and 70 lines in each HR14B spectrum; however, significant variation was seen as a function of $\mathrm{S} / \mathrm{N}$ and the effective temperature of the target. In the majority of cases the line-by-line variance for each individual radial velocity estimate was in the range $0.5 \lesssim \sigma \lesssim 1.5 \mathrm{~km} \mathrm{~s}^{-1}$; we conservatively adopted this quantity to represent the uncertainty on each such measurement.

While checking our results we noticed the presence of small systematic offsets between velocities measured for a given star from different settings. The $2 \sigma$-clipped mean offsets were $V_{\mathrm{HR} 13}-V_{\mathrm{HR} 11}=0.55 \mathrm{~km} \mathrm{~s}^{-1}$ (97 stars) and $V_{\mathrm{HR} 13}-V_{\mathrm{HR} 14 \mathrm{~B}}=$ $-0.36 \mathrm{~km} \mathrm{~s}^{-1}$ (94 stars). The origin of these offsets is not clear, but they were straightforward to correct. For each setting we precisely measured the positions of all the atmospheric emission features in each of the 16 blank sky spectra. We then matched these measurements against the wavelengths listed in the atlas of Osterbrock et al. (1996) and derived the mean (2.5 $\sigma$-clipped) offset. We found that the HR11 spectra needed to be shifted by $0.39 \mathrm{~km} \mathrm{~s}^{-1}$, the HR13 spectra by $-0.17 \mathrm{~km} \mathrm{~s}^{-1}$, and the HR14B spectra by $-0.52 \mathrm{~km} \mathrm{~s}^{-1}$. No dependence on fiber number (i.e., the position of individual sky spectra on the FLAMES CCD) was evident. Applying these corrections almost completely removed the mean systematic offsets between velocities measured from the three different settings for a given target. We combined these corrected quantities in a weighted average to obtain a final radial velocity for each star, along with its associated uncertainty.

\section{CLUSTER MEMBERSHIP}

\subsection{Stars within the Truncation Radius}

The upper panel of Figure 4 shows radial velocity versus projected distance from the center of NGC 1846 for all 29 ACS stars and 76 MCPS stars. We adopted $\alpha=05: 07: 33.66$, $\delta=-67: 27: 40.7$ for the cluster center, determined using our ACS photometry in the FK5 astrometric frame. The tight grouping of objects to the lower left of the plot is indicative of the cluster. To determine membership we imposed a maximum allowed radius of $161^{\prime \prime}$, corresponding to the truncation radius measured for NGC 1846 by Goudfrooij et al. (2009). We also calculated the mean velocity $\bar{V}$ of targets lying within the halflight radius of $r_{h}=34$. 5 obtained by the same authors, on the basis that these objects are the most likely to be cluster members, and then imposed a generous radial velocity cut of $\pm 10 \mathrm{~km} \mathrm{~s}^{-1}$ about this value. ${ }^{4}$ These criteria resulted in the exclusion of seven ACS stars, with one additional star lying on the boundary of the allowed region of parameter space. The lower panel of Figure 4 shows a close-up of this region. Although the candidate star has a radial velocity only $\approx 10 \mathrm{~km} \mathrm{~s}^{-1}$ higher than the systemic velocity of NGC 1846, this corresponds to more than five times the velocity dispersion at this radius within the cluster (see Section 4 below) and we therefore also excluded this object.

Our 21 probable stellar members of NGC 1846 are listed in Table 2 along with their ACS/WFC photometry and measured radial velocities. Note, from the upper panel of Figure 4, that the velocity of the cluster overlaps significantly with the range measured for non-members (i.e., those objects outside the truncation radius). Thus, we cannot be certain that our assumed sample is entirely free of field interlopers. To assess the likelihood of this, we calculated an indicative membership probability for all 21 stars in our sample. For each object, we used the cluster surface density profile of Goudfrooij et al. (2009; see the beginning of Section 4, below) to estimate the likelihood that the star under consideration could be a nonmember based on the relative densities of the cluster and field at the appropriate radius. We then counted how many of the 76 MCPS stars from outside $r_{t}$ lay within the interval $\bar{V} \pm\left|v_{i}+\sigma_{i}\right|$, where $v_{i}$ and $\sigma_{i}$ are the velocity and associated uncertainty

\footnotetext{
4 We note that according to the 2010 update of the Harris (1996) online catalog, all but the most massive Galactic globular clusters have central velocity dispersions of only a few $\mathrm{km} \mathrm{s}^{-1}$ (see also Lane et al. 2009, 2010a, 2010b).
} 
Table 2

Data on the Radial Velocity Members of NGC 1846 Studied in This Work

\begin{tabular}{|c|c|c|c|c|c|c|c|c|c|}
\hline \multirow{2}{*}{$\begin{array}{l}\text { Target } \\
\text { Name }\end{array}$} & \multicolumn{2}{|c|}{ Position (J2000.0) } & \multirow{2}{*}{$\begin{array}{c}\text { Radius } \\
\left({ }^{\prime \prime}\right)\end{array}$} & \multirow{2}{*}{$\begin{array}{l}\text { P.A. } \\
\left({ }^{\circ}\right)\end{array}$} & \multirow[t]{2}{*}{$B$} & \multirow[t]{2}{*}{$V$} & \multirow[t]{2}{*}{$I$} & \multirow{2}{*}{$\begin{array}{l}\text { Velocity } \\
\left(\mathrm{km} \mathrm{s}^{-1}\right)\end{array}$} & \multirow[t]{2}{*}{$P_{\text {mem }}$} \\
\hline & R.A. & Decl. & & & & & & & \\
\hline ACS-001 & 050736.8 & -672745.9 & 19.0 & 105.8 & 18.16 & 16.56 & 14.86 & $236.1 \pm 0.5$ & 0.996 \\
\hline ACS-013 & 050733.6 & -672641.2 & 59.5 & 359.6 & 18.35 & 16.99 & 15.56 & $238.1 \pm 0.5$ & 0.995 \\
\hline ACS-017 & 050738.4 & -672811.7 & 41.2 & 138.7 & 18.51 & 17.18 & 15.73 & $236.6 \pm 0.4$ & 0.990 \\
\hline ACS-025 & 050736.2 & -672758.8 & 23.2 & 141.2 & 18.63 & 17.28 & 15.93 & $238.3 \pm 0.5$ & 0.999 \\
\hline ACS-030 & 050739.0 & -672823.2 & 52.5 & 144.1 & 18.66 & 17.43 & 16.12 & $240.7 \pm 0.6$ & 0.985 \\
\hline ACS-036 & 050730.4 & -672935.7 & 116.5 & 189.4 & 18.86 & 17.63 & 16.38 & $238.7 \pm 0.5$ & 0.988 \\
\hline ACS-043 & 050721.6 & -672725.3 & 70.9 & 282.5 & 18.84 & 17.70 & 16.45 & $239.7 \pm 0.5$ & 0.994 \\
\hline ACS-046 & 050732.6 & -672745.5 & 7.6 & 230.5 & 19.11 & 17.92 & 16.68 & $237.5 \pm 0.6$ & 0.997 \\
\hline ACS-047 & 050734.5 & -672824.2 & 43.7 & 173.7 & 19.02 & 17.84 & 16.62 & $239.2 \pm 0.7$ & 0.997 \\
\hline ACS-051 & 050736.6 & -672733.5 & 18.4 & 66.8 & 19.10 & 17.96 & 16.71 & $235.6 \pm 0.8$ & 0.992 \\
\hline ACS-053 & 050729.6 & -672627.4 & 77.0 & 342.3 & 19.09 & 17.89 & 16.65 & $242.9 \pm 0.8$ & 0.919 \\
\hline ACS-059 & 050728.6 & -672844.8 & 70.3 & 204.3 & 19.15 & 18.04 & 16.85 & $237.8 \pm 0.8$ & 0.975 \\
\hline ACS-066 & 050749.6 & -672901.4 & 121.9 & 131.5 & $\ldots$ & 18.18 & 17.00 & $239.4 \pm 0.8$ & 0.988 \\
\hline ACS-081 & 050730.4 & -672804.2 & 30.1 & 218.8 & 18.45 & 17.10 & 15.74 & $239.2 \pm 0.4$ & 0.998 \\
\hline ACS-082 & 050730.1 & -672727.4 & 24.5 & 303.1 & 18.47 & 17.14 & 15.81 & $243.4 \pm 0.6$ & 0.984 \\
\hline ACS-085 & 050730.0 & -672642.7 & 61.7 & 340.1 & 18.53 & 17.15 & 15.81 & $238.2 \pm 0.6$ & 0.995 \\
\hline ACS-090 & 050730.2 & -672746.1 & 20.8 & 254.9 & 18.76 & 17.53 & 16.29 & $238.0 \pm 0.6$ & 0.998 \\
\hline ACS-092 & 050728.7 & -672824.4 & 52.2 & 213.3 & 18.75 & 17.57 & 16.33 & $238.2 \pm 0.6$ & 0.996 \\
\hline ACS-102 & 050727.4 & -672927.6 & 112.7 & 198.6 & 18.97 & 17.90 & 16.75 & $238.5 \pm 0.6$ & 0.988 \\
\hline ACS-112 & 050743.3 & -672649.9 & 75.2 & 47.5 & $\ldots$ & 18.02 & 16.90 & $239.8 \pm 0.7$ & 0.993 \\
\hline Мо-17 & 050725.3 & -672851.0 & 83.5 & 214.3 & $\ldots$ & $\ldots$ & $\ldots$ & $236.7 \pm 1.5$ & 0.967 \\
\hline
\end{tabular}

for the star under consideration, and used this information to estimate the likelihood that the star could be a non-member based on the deviation of its velocity from the cluster mean. We defined the final membership probability for the star, $P_{\mathrm{mem}}$, to be the complement of the product of this quantity and that estimated from the density profile.

The values of $P_{\mathrm{mem}}$ are listed in Table 2. Note that these are indicative lower limits only. They are subject to stochastic fluctuations due to the small number of MCPS objects lying comparably near to the cluster mean velocity, and do not include any information about the photometric selection criteria we employed in defining our initial sample of targets (Figure 1) which would effectively reduce the number of viable MCPS stars within the allowed velocity intervals by a factor $\gtrsim 2$.

The membership probabilities are very high for all objects. The most likely non-member is ACS-053, which has a membership probability of $\approx 92 \%$; for most others, $P_{\text {mem }} \gtrsim 99 \%$. These simple calculations give us confidence that we have defined a clean set of NGC 1846 members. We consider the remainder of our ACS and MCPS targets to belong to the LMC field (or, possibly, the Galactic foreground); further study of these objects is beyond the scope of the present work.

In order to aid future observers of NGC 1846 we list, in Table 3, those stars lying within the truncation radius of $161^{\prime \prime}$ which are not radial velocity members of the cluster. Members and non-members are also distinguished on the CMDs in Figure 1.

\subsection{The Planetary Nebula Mo-17}

We briefly consider the planetary nebula Mo-17 in more detail. This object was first cataloged by Morgan (1994), and, based on its proximity to NGC 1846 (it lies at a radius of 83 ".5 $\approx 20.3 \mathrm{pc}$ ), Kontizas et al. (1996) suggested that it might in fact belong to the cluster. The catalog of Reid \& Parker (2006) lists a radial velocity of $241.8 \pm 20.0 \mathrm{~km} \mathrm{~s}^{-1}$, which is consistent with literature estimates for the motion of NGC 1846.
Table 3

Data on Non-members of NGC 1846

\begin{tabular}{lcccc}
\hline \hline Target & \multicolumn{2}{c}{ Position (J2000.0) } & Radius & $\begin{array}{c}\text { Velocity } \\
\text { Name }\end{array}$ \\
\cline { 2 - 3 } & R.A. & Decl. & $\left.\mathrm{km} \mathrm{s}^{-1}\right)$ \\
\hline ACS-019 & 050714.5 & -672816.4 & 115.8 & $278.7 \pm 0.5$ \\
ACS-022 & 050720.0 & -672936.9 & 140.4 & $309.9 \pm 0.7$ \\
ACS-024 & 050721.1 & -672701.3 & 82.1 & $249.1 \pm 0.5$ \\
ACS-026 & 050723.6 & -672650.6 & 76.6 & $262.8 \pm 0.5$ \\
ACS-029 & 050714.7 & -672915.8 & 144.8 & $279.5 \pm 0.5$ \\
ACS-054 & 050726.3 & -672656.8 & 61.2 & $292.0 \pm 0.8$ \\
ACS-070 & 050710.5 & -672831.1 & 142.1 & $281.0 \pm 0.8$ \\
ACS-072 & 050714.4 & -672748.1 & 111.1 & $291.0 \pm 0.9$ \\
\hline
\end{tabular}

Figure 5 shows ACS/WFC thumbnails of Mo-17 in the F435W, F555W, and F814W passbands. ${ }^{5}$ The nebula is circular in appearance, with a diameter of approximately 1 1". $3 \approx 0.3 \mathrm{pc}$. It is brightest in the F555W image, in which it also appears to have two distinct lobes, or perhaps an outer ring. The central star may be visible - there is a blend of two objects lying near the middle of the nebula. The fainter of these (the upper object in Figure 5) is much bluer than the brighter; however, due to their close proximity, precise photometry is very difficult.

As noted previously, we allocated a MEDUSA fiber to Mo- 17 . The fiber diameter of 1".2 corresponds well to the size of the nebula. Example spectra from the HR13 and HR14B settings are plotted in Figure 6. Although there is sparse contamination from sky line residuals, in the HR 13 setting [O I] $\lambda 6300$ emission is clearly visible, while in the HR14B spectrum there is strong emission from the [N II] $\lambda 6548$ and $\lambda 6583$ lines and $\mathrm{H} \alpha$.

\footnotetext{
5 Note that Shaw et al. (2006) have previously reported on archival HST/ACS imaging of this object; however, based on the coordinates and image they present, a nearby face-on background spiral galaxy was apparently misidentified as the planetary nebula. It is not clear whether their brief description of the appearance of Mo-17 corresponds to the correct object or to this interloper.
} 

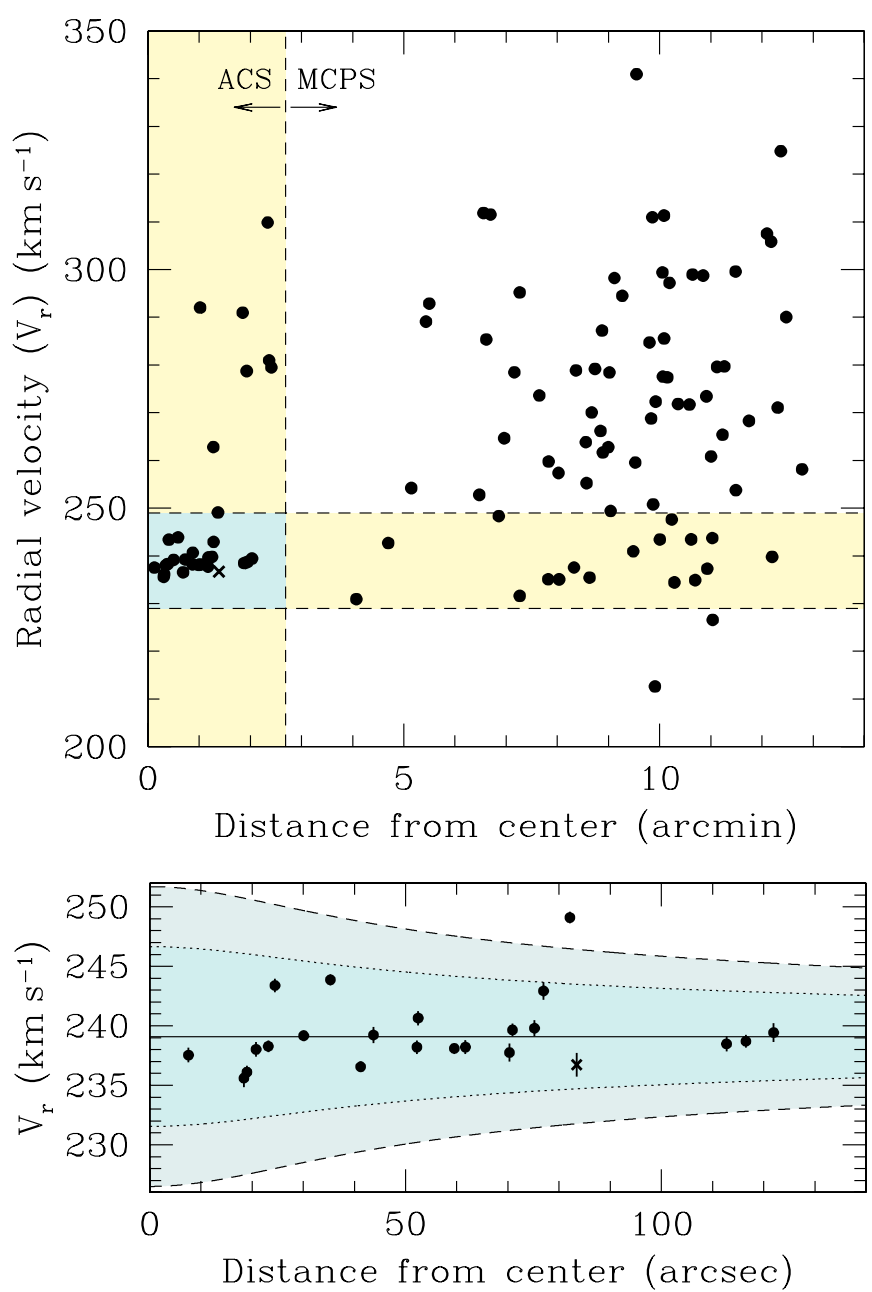

Figure 4. Radial velocity vs. distance from the center of NGC 1846 for all of our FLAMES science targets. The upper panel spans the full radial coverage, and the full range of radial velocities. The vertical shaded area indicates the region lying inside the nominal boundary of the cluster, while the horizontal shaded region denotes our initial radial velocity cut of $\pm 10 \mathrm{~km} \mathrm{~s}^{-1}$ about the mean velocity of targets lying within $r_{h}=34$.'5. The lower panel zooms in on the intersection of these two regions. Here, the shaded areas indicate \pm 5 and \pm 3 times the velocity dispersion at given radius, calculated according to Equation (4) (see also Figure 10). In both panels the position of the planetary nebula Mo-17 is marked with a cross.

(A color version of this figure is available in the online journal.)
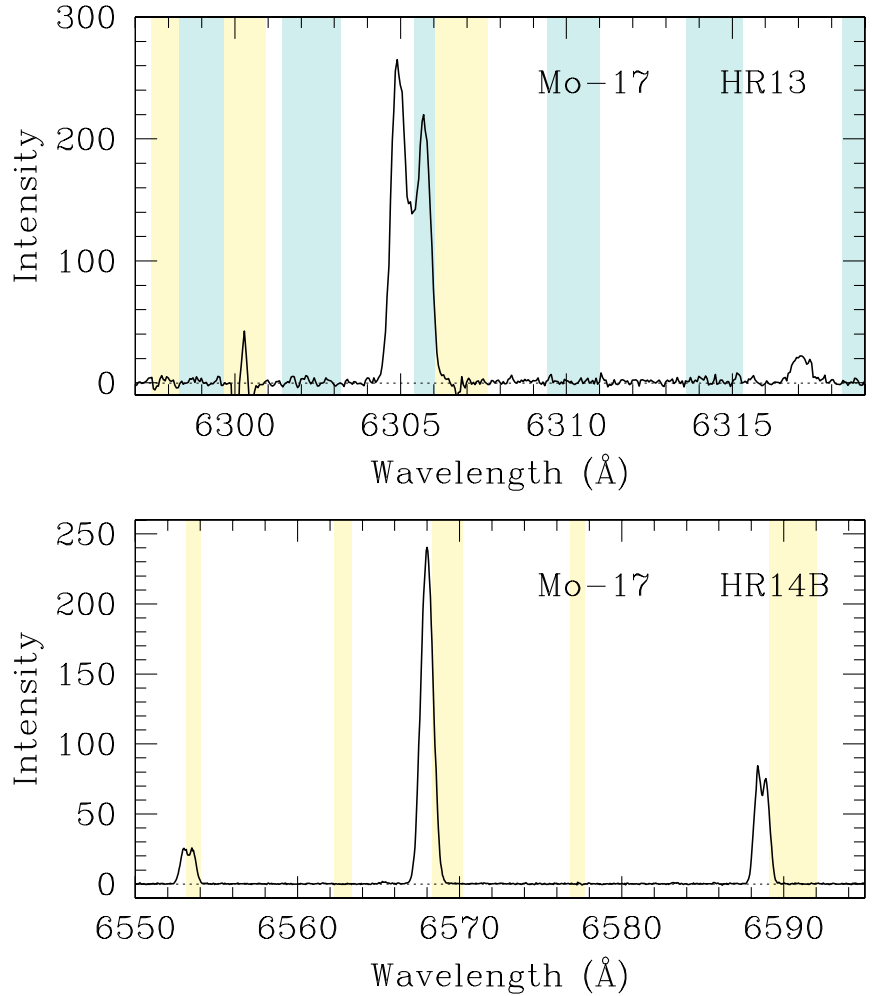

Figure 6. Example HR13 and HR14B spectral segments for the NGC 1846 planetary nebula Mo-17. As in Figure 3 these have been corrected to the heliocentric frame, but no correction for the radial velocity of the target has been made. Note that the intensity scale is in arbitrary units. We have subtracted the sky lines as accurately as possible across these small segments. As before, regions in the sky line mask are shaded yellow. Note that most lines have subtracted cleanly; however strong residuals are present for the [O I] line near $\lambda 6300$. Regions masked due to the presence of telluric absorption are shaded blue. The HR13 panel spans $\sim 22 \AA$; nebular [O I] emission is visible at $\lambda 6305$; note that this line is broadened and double-peaked. Weak [S III] emission is also visible near $\lambda 6317$. The HR14B panel spans a larger spectral range of $\sim 45 \AA$. Double-peaked [N II] emission lines are visible at either end of the segment, with a strong single-peaked $\mathrm{H} \alpha$ line near $\lambda 6568$. This line was used to derive the velocity of Mo-17.

(A color version of this figure is available in the online journal.)
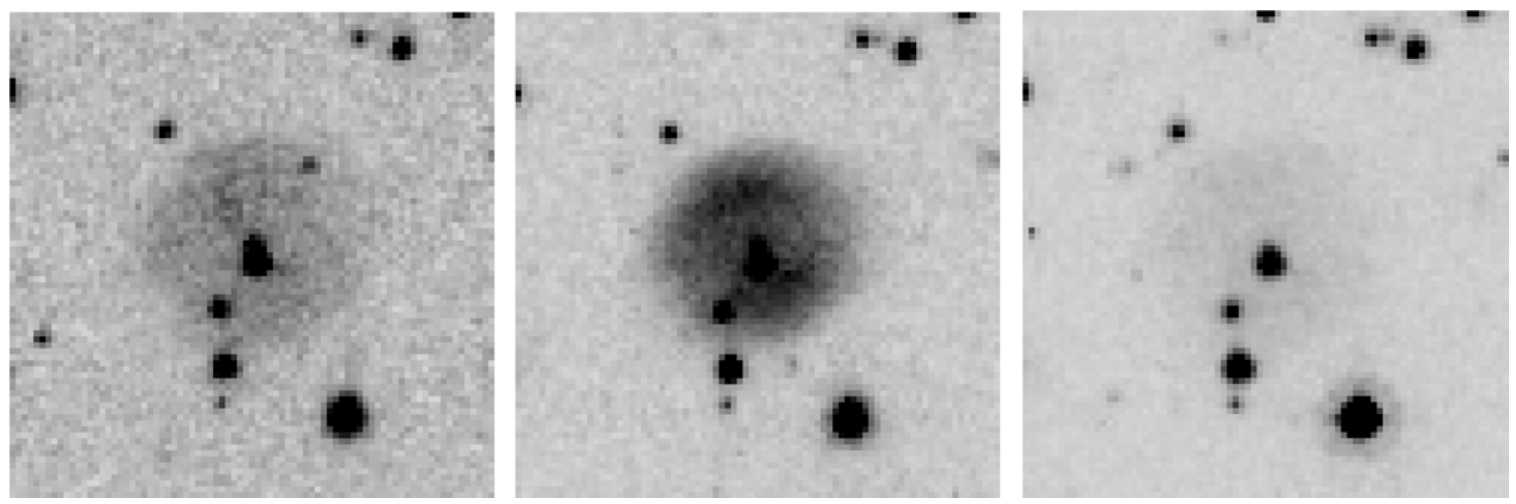

Figure 5. HST ACS/WFC images of the NGC 1846 planetary nebula Mo-17-from left to right, taken with the F435W, F555W, and F814W filters. Each thumbnail is $4^{\prime \prime}$ on a side. 
Also detected in the HR13 spectrum is a weak [S III] $\lambda 6312$ line, and the $\left[\mathrm{O}_{\mathrm{I}}\right] \lambda 6363$ line (not plotted). The HR11 setting (not shown) covers only one weak line, [N II] $\lambda 5755$, while the LR02 spectrum (also not shown) exhibits a variety of emission features including the Balmer lines $\mathrm{H} \gamma, \mathrm{H} \delta$ and $\mathrm{H} \epsilon$, several $\mathrm{HeI}$ lines, [O III] $\lambda 4363$, and $[\mathrm{Ne} \mathrm{III]} \lambda 3967$.

In Figure 6 is it quite evident that the strong [O I] $\lambda 6300$ and $[\mathrm{N}$ II $] \lambda \lambda 6548,6583$ lines are broad and double-peaked. The [O I] $\lambda 6363$ line also follows this pattern. It is likely that this reflects the lobed structure seen in the F555W image in Figure 5. The F555W filter spans the range $\sim 4700-6100 \AA$, within which emission from [O III] $\lambda 4959$ and $\lambda 5007$ will be dominant. The separation of the peaks in the two [O I] lines indicates outflow velocities of approximately $\pm 20 \mathrm{~km} \mathrm{~s}^{-1}$, while the [N II] peaks suggest $\pm 10 \mathrm{~km} \mathrm{~s}^{-1}$.

The velocity structure present in these emission lines makes it difficult to obtain a precise estimate for the radial velocity of Mo-17. Notably however, the $\mathrm{H} \alpha$ line, while equivalently broad as the lines discussed above, has only a single peak and is highly symmetric. $\mathrm{H} \alpha$ falls between the wavelength coverage of the ACS/WFC F555W and F814W filters. However, light in the F435W image of the nebula appears much more evenly distributed than in F555W; we know from our LR02 spectra that emission in the range covered by this filter $(\sim 3700-4800 \AA)$ comes predominantly from the Balmer series. Given this, and the circular symmetry of the nebula, we make the assumption that the peak of the $\mathrm{H} \alpha$ line reflects the overall radial motion of Mo-17, and derive a heliocentric velocity $V_{r}=236.7 \mathrm{~km} \mathrm{~s}^{-1}$. Estimating the mid-points of the two [O I] and two [N II] lines and taking the average of these measurements leads to a velocity within $\approx 1.2 \mathrm{~km} \mathrm{~s}^{-1}$ of this, and we thus adopt an overall uncertainty of $\pm 1.5 \mathrm{~km} \mathrm{~s}^{-1}$ on our result. As plotted in Figure 4, Mo-17 is very likely a member of NGC 1846; the indicative membership probability, $P_{\text {mem }} \approx 97 \%$. We include this object in Table 2 .

\section{CLUSTER KINEMATICS}

Our radial velocity measurements allowed us to investigate the internal dynamics of NGC 1846. In what follows we make use of the cluster structural parameters measured by Goudfrooij et al. (2009). These authors fit a King (1962) model,

$$
n\left(r_{p}\right)=n_{0}\left[\frac{1}{\sqrt{1+\left(r_{p} / r_{c}\right)^{2}}}-\frac{1}{\sqrt{1+\left(r_{t} / r_{c}\right)^{2}}}\right]^{2},
$$

to a radial number-density profile constructed from their ACS/WFC imaging of the cluster. They found a core radius $r_{c}=26^{\prime \prime} .0$ and a truncation radius $r_{t}=161^{\prime \prime} .2$. They also measured $r_{h}=34^{\prime \prime} .5$-assuming that the cluster is not strongly segregated by luminosity (or mass), this is a good estimate of its projected half-light (or half-mass) radius. Adopting the canonical LMC distance modulus $\mu=18.5$, these values correspond to $r_{c}=6.3 \mathrm{pc}, r_{h}=8.4 \mathrm{pc}$, and $r_{t}=39.2 \mathrm{pc}$.

\subsection{Rotation}

We first checked for any signature of rotation within the cluster, following a commonly used procedure (see, e.g., Lane et al. 2009, 2010a, 2010b; Bellazzini et al. 2012, and references therein). We calculated the position angle (P.A., east of north) of each member in the plane of the sky with respect to the cluster center, then split the sample with the dividing line P.A. $=0^{\circ}-180^{\circ}$ and calculated the difference in mean radial
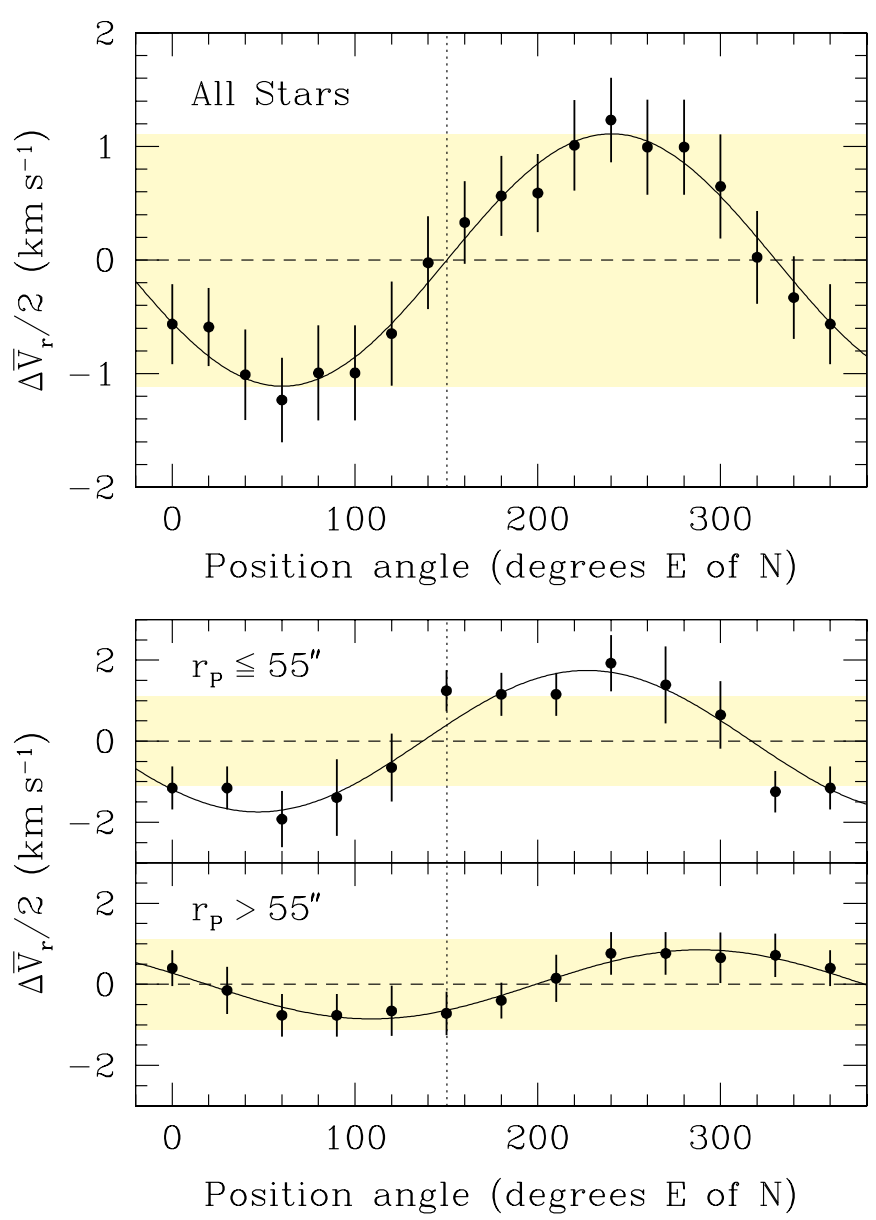

Figure 7. Plots of $\Delta \bar{V}_{r} / 2$ as a function of the position angle of the dividing line for stars in NGC 1846. The upper panel shows the measurements and best-fitting model for the entire sample - note the clean sinusoidal shape of the rotation curve. The shaded region indicates the measured amplitude of rotation, $A_{\text {rot }}=1.1 \mathrm{~km} \mathrm{~s}^{-1}$. In the lower two panels we divide the sample in half using a radius $r_{p}=55^{\prime \prime}$ and repeat the measurements. The inner half of the sample appears to rotate much more strongly than the outer half. For comparison to the upper panel, the shaded region again denotes the amplitude of rotation measured for the full sample.

(A color version of this figure is available in the online journal.)

velocity between the two subgroups of stars $\left(\Delta \bar{V}_{r}\right)$. This process was then repeated with the position angle of the dividing line incremented by $20^{\circ}$ each time.

If coherent rotation is present in the cluster and does not lie entirely in the plane of the sky, this should be manifested in the form of a sinusoidal pattern when $\Delta \bar{V}_{r}$ is plotted as a function of the position angle of the dividing line. The position angle at which the maximum amplitude of this sinusoid occurs corresponds to the projected axis of rotation, while the amplitude itself represents twice that of the mean rotation-i.e., $A_{\text {rot }}=\Delta \bar{V}_{r} / 2$. The observed (projected) amplitude $A_{\text {rot }}$ is a lower limit to the true amplitude of rotation $A_{\text {true }}$ as there is a correction factor $\sin i$ to consider, where $i$ is the inclination of the rotation axis with respect to the plane of the sky $\left(i=90^{\circ}\right.$ represents an "edge-on" cluster, where the observed amplitude of rotation would match the true amplitude, while $i=0^{\circ}$ is a pole-on cluster where no rotation would be seen from radial velocity measurements irrespective of the true amplitude).

The upper panel of Figure 7 shows the results for our sample of 22 tracers in NGC 1846. We plot $\Delta \bar{V}_{r} / 2$ in order to more clearly elucidate the observed rotation amplitude. To determine 
the point-to-point uncertainties, we used a bootstrapping method whereby we generated a large number of mock systems using our observed sample. Each mock system consisted of 22 stellar velocities chosen randomly from our 22 measurements, with repeated selection allowed. Every time we selected a velocity $v_{i}$ we also generated a random Gaussian deviate of $v_{i}$ (that is, a value from a Gaussian distribution with $\sigma_{i}$-i.e., the measurement uncertainty in $v_{i}$ ) and added this to the velocity. We then repeated our measurement of $\Delta \bar{V}_{r}$ as a function of position angle for all mock systems, with the distribution of measurements at a given P.A. indicative of the uncertainty at that point. This procedure allowed us to account naturally for both the (relatively small) size of our sample and the individual uncertainties on our measured radial velocities.

Our results show a clear sinusoidal pattern, suggestive that systemic rotation is present in NGC 1846. The weighted best fit has $A_{\text {rot }}=1.1 \mathrm{~km} \mathrm{~s}^{-1}$ and the axis of rotation lying along the line P.A. $=60^{\circ}-240^{\circ}$. Uncertainties derived directly from this fit likely underestimate the true uncertainties because the points are all correlated. Bellazzini et al. (2012) adopt $\pm 0.5 \mathrm{~km} \mathrm{~s}^{-1}$ and $\pm 30^{\circ}$ as conservative $1 \sigma$ uncertainties on their measured rotation amplitudes and axis orientations, based on experimentation with subsamples of their observed stars and comparisons with external samples. We are not in a position to conduct similar tests; instead we investigated this problem using another large set of mock systems.

The question is how strongly we can constrain the amplitude and orientation of rotation in the cluster given the sample of stars we have measured. To this end each mock system consisted of 22 stars at the same radius and P.A. as in the real sample. We assumed simple cylindrical rotation with amplitude and orientation as measured above-this defined a velocity for each star based on its P.A., to which we added a random measurement uncertainty as previously, and a second random component defined by the velocity dispersion profile of NGC 1846 (see Section 4.2, below). We then followed the same procedure outlined above to measure the rotation amplitude and orientation, and examined the distributions of these recovered quantities over all mock systems to determine our uncertainties. We found the measured amplitude of rotation to be uncertain at the $\pm 0.4 \mathrm{~km} \mathrm{~s}^{-1}$ level, and the orientation of the rotation axis to be uncertain at the $\pm 20^{\circ}$ level-similar to the values adopted by Bellazzini et al. (2012).

We next decided to test whether there was any change in the degree of rotation with radius within the cluster. To do this we split our sample in half using a dividing radius $r_{p}=55^{\prime \prime}$, which corresponds to roughly 1.5 times the half-light radius, and repeated our measurements using only stars within, and then outwith, this radius. The results are shown in the lower panel of Figure 7. Although the samples are by now rather small, there is an indication that the rotation amplitude for the inner sample is significantly larger than that for the outer sample-the best fits have amplitudes of $1.8 \pm 0.6 \mathrm{~km} \mathrm{~s}^{-1}$ and $0.8 \pm 0.4 \mathrm{~km} \mathrm{~s}^{-1}$ respectively. The P.A. of the rotation axis changes by only a small amount for the inner sample, to $47 \pm 25^{\circ}$, but increases substantially for the outer sample, to $109 \pm 35^{\circ}$. The quoted uncertainties were again derived using large sets of mock systems.

Bellazzini et al. (2012) note that variation of rotation amplitude with radius is not unusual in Galactic globular clusters, such that the rotation amplitude determined using stars at all radii typically underestimates the maximum rotation amplitude in the cluster. Their experiments indicate that $\Delta \bar{V}_{r}=2 A_{\text {rot }}$,
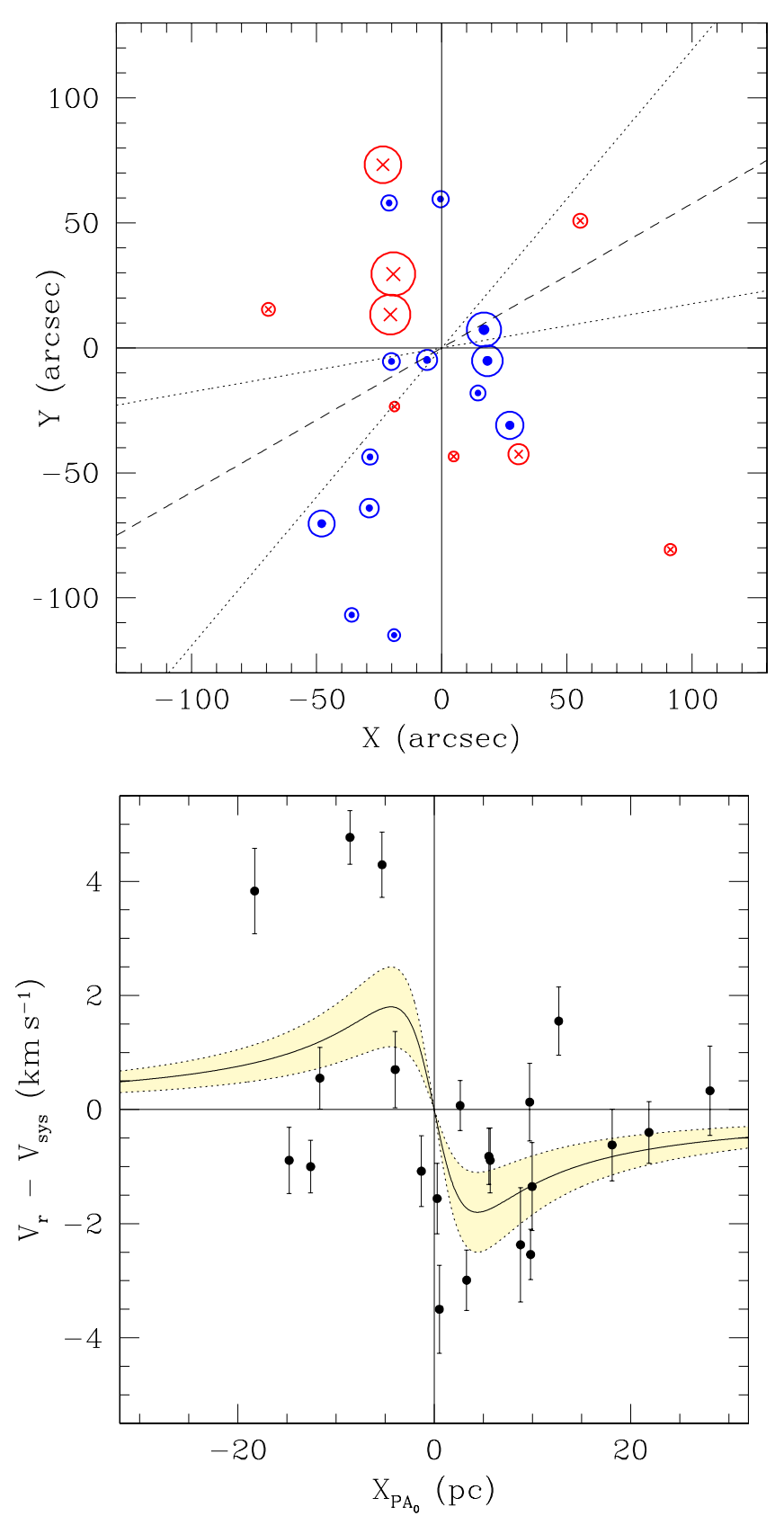

Figure 8. Additional evidence for systemic rotation in NGC 1846. The upper panel shows a tangent plane projection of the positions and velocities of the measured stars within the cluster. Red crossed points (alternatively blue dotted points) indicate stars with velocities greater (less) than the global mean velocity. The point sizes are proportional to the deviation of the velocity from the mean. The preferred axis of rotation, oriented at a position angle $60 \pm 20^{\circ}$ east of north, is marked. In the lower panel we plot a rotation curve for NGC 1846, where $X_{\mathrm{PA}_{0}}$ is the perpendicular distance of from the rotation axis and we have assumed an LMC distance modulus $\mu=18.5$. The best fitting model, of the form of Equation (2) is marked, along with the curves represented by $\pm 1 \sigma$ on the maximum amplitude (see the text).

(A color version of this figure is available in the online journal.)

where $A_{\text {rot }}$ is calculated (as we have done here) using stars at all radii, is likely to be a better estimate of the maximum rotation amplitude. This is consistent with the lower panel of Figure 7, which suggests that the maximum projected rotation amplitude for NGC 1846 could be as high as $\approx 2 \mathrm{~km} \mathrm{~s}^{-1}$.

As a qualitative check on our detection of rotation in NGC 1846 we used the best-fit solution derived from the full sample to construct a rotation curve. The top panel of Figure 8 


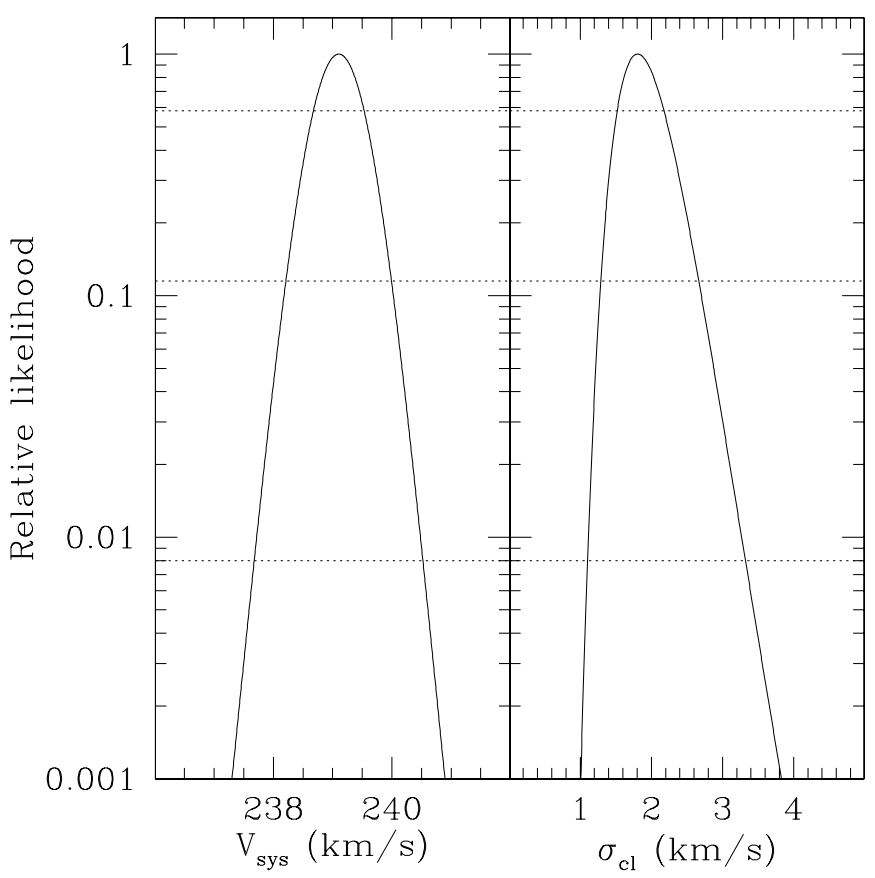

Figure 9. Relative likelihood distributions for the systemic velocity $V_{\text {sys }}$ and the mean velocity dispersion $\sigma_{\mathrm{cl}}$, where in each case we have marginalized with respect to the other variable. The dashed horizontal lines indicate the levels corresponding to $1 \sigma, 2 \sigma$, and $3 \sigma$ uncertainties.

shows a tangent plane projection of the positions and velocities of our measured members within the cluster. The preferred axis of rotation, lying along the line P.A. $=60^{\circ}-240^{\circ} \pm 20^{\circ}$, is indicated. In the lower panel we plot, for each star, the offset from the cluster systemic velocity (derived in Section 4.2 below) as a function of the perpendicular distance from the rotation axis $\left(X_{\mathrm{PA}_{0}}\right)$. Bearing in mind that almost all of the point-to-point scatter can be accounted for by the cluster velocity dispersion (which is of the order of $2 \mathrm{~km} \mathrm{~s}^{-1}$-again, see Section 4.2 below), these plots clearly support the detection of systemic rotation in NGC 1846. As an indicative measure, we fit a rotation curve of the form (as in, e.g., Lynden-Bell 1967; Gott 1973; Hénault-Brunet et al. 2012a)

$$
V_{\text {rot }}=\frac{2 A_{\text {rot }}}{r_{\text {peak }}} \times \frac{X_{\mathrm{PA}_{0}}}{1+\left(X_{\mathrm{PA}_{0}} / r_{\text {peak }}\right)^{2}},
$$

where $r_{\text {peak }}$ is the projected radius at which the peak amplitude of rotation occurs. The best fitting model has $A_{\text {rot }}=1.8 \pm$ $0.7 \mathrm{~km} \mathrm{~s}^{-1}$ and $r_{\text {peak }}=4.4 \pm 3.1 \mathrm{pc}$. Although not particularly strongly constrained, these results are entirely consistent with those from our initial analysis above.

\subsection{Mean Velocity and Velocity Dispersion}

Next, we corrected the velocity of each of our tracers for the systemic rotation, and calculated the global mean velocity and velocity dispersion, along with the velocity dispersion as a function of distance from the cluster center. To make the rotation corrections, we used the best fit to the full-sample rotation curve (i.e., the top panel of Figure 7). Although, as discussed, we suspect that the degree of rotation does vary as a function of radius within the cluster, our sample of kinematic probes is not sufficiently large to accurately measure this variation; the use of the mean rotation curve represents an adequate compromise. In any case, we note that even the extreme case of applying

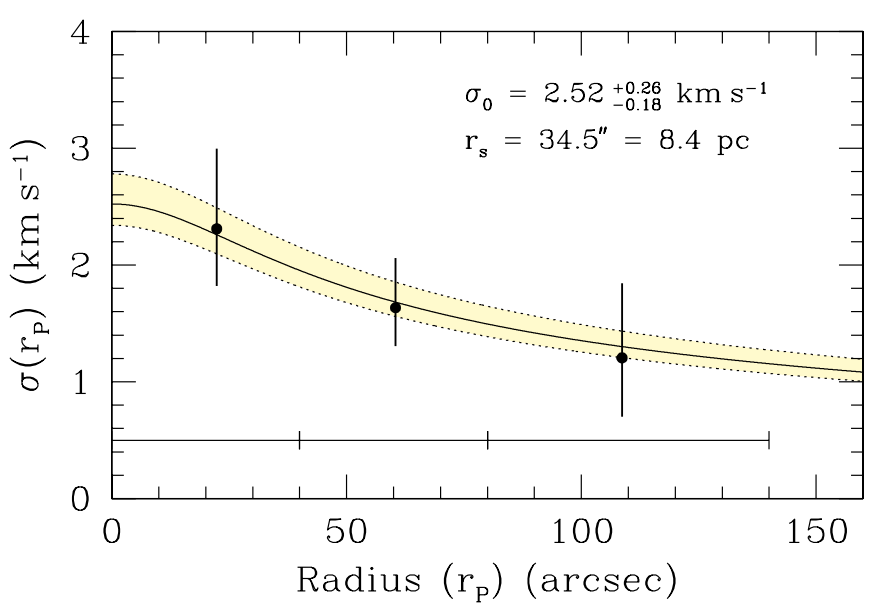

Figure 10. Our indicative three-point velocity dispersion profile for NGC 1846, together with the best-fitting Plummer model described in the text. The shaded region corresponds to the $1 \sigma$ uncertainties on the central dispersion $\sigma_{0}$.

(A color version of this figure is available in the online journal.)

no correction for rotation changes the global mean velocity by less than the uncertainty on the measurement, and the velocity dispersion results by less than $\sim 15 \%$.

To calculate the mean radial velocity and velocity dispersion for NGC 1846 we used a maximum likelihood technique following that defined by Walker et al. (2006). We assume that the measured velocities for our stars, denoted $\left\{v_{1}, \ldots, v_{N}\right\}$, are normally distributed about the systemic velocity $V_{\text {sys }}$ according to the associated measurement uncertainties $\sigma_{i}$ and the intrinsic cluster velocity dispersion $\sigma_{\mathrm{cl}}$. We can obtain numerical estimates for the quantities $V_{\text {sys }}$ and $\sigma_{\mathrm{cl}}$ by maximizing the logarithm of the joint probability function for $\left\{v_{1}, \ldots, v_{N}\right\}$-i.e.,

$$
\begin{aligned}
\ln (p)= & -\frac{1}{2} \sum_{i=1}^{N} \ln \left(\sigma_{i}^{2}+\sigma_{\mathrm{cl}}^{2}\right)-\frac{1}{2} \sum_{i=1}^{N} \frac{\left(v_{i}-V_{\mathrm{sys}}\right)^{2}}{\left(\sigma_{i}^{2}+\sigma_{\mathrm{cl}}^{2}\right)} \\
& -\frac{1}{2} N \ln (2 \pi) .
\end{aligned}
$$

Figure 9 shows the relative likelihood of both $V_{\text {sys }}$ and $\sigma_{\mathrm{cl}}$, where in each case we have marginalized with respect to the other variable. To determine $1 \sigma$ uncertainties we calculated the parameter limits for the region containing the central $68.3 \%$ of the distribution function.

We find a systemic velocity for NGC 1846 of $V_{\text {sys }}=239.1 \pm$ $0.4 \mathrm{~km} \mathrm{~s}^{-1}$. This is consistent with the value of $240 \pm 10 \mathrm{~km} \mathrm{~s}^{-1}$ determined by Olszewski et al. (1991), but slightly larger than that of $235.2 \pm 0.9 \mathrm{~km} \mathrm{~s}^{-1}$ from Grocholski et al. (2006). For the mean velocity dispersion we find $\sigma_{\mathrm{cl}}=1.81_{-0.29}^{+0.37} \mathrm{~km} \mathrm{~s}^{-1}$. A value of zero is excluded at $\gg 5 \sigma$, indicating that we have resolved the intrinsic cluster dispersion.

The kinematic properties of globular clusters are commonly framed in terms of the central velocity dispersion, $\sigma_{0}$. Given that we have members extending to $\approx 4 r_{h}$ it is likely that our measurement of $\sigma_{\mathrm{cl}}$ is an underestimate of this quantity. To assess this we recalculated the velocity dispersion as a function of cluster radius. Because of our relatively small sample size we are restricted to just three radial bins, corresponding roughly to stars inside $r_{h}$, stars between $r_{h}$ and twice $r_{h}$, and stars outside $2 r_{h}$. For each bin we utilized Equation (3) as before, but with fixed $V_{\text {sys }}=239.1 \mathrm{~km} \mathrm{~s}^{-1}$.

Our results are shown in Figure 10. A marginally significant decrease in the velocity dispersion with radius is evident. Even 
in the outermost bin the dispersion is resolved (i.e., a value of zero is excluded at $\mathrm{a} \approx 3 \sigma$ level). As an indicative measure we assume NGC 1846 to be isotropic and fit a projected Plummer (1911) model to our three-point dispersion profile:

$$
\sigma^{2}\left(r_{p}\right)=\frac{\sigma_{0}^{2}}{\sqrt{1+r_{p}^{2} / a^{2}}}
$$

Here $a$ is a scale radius which, for the family of projected Plummer (1911) models, is equal to the half-light (half-mass) radius $r_{h}$ if the mass-to-light ratio $(M / L)$ is constant within the cluster. Note that we are not asserting here that a Plummer model is the most appropriate model to describe the internal kinematics of NGC 1846-with our poor radial resolution we are not in a position to undertake such an analysis. Rather, we use the Plummer model as a convenient parameterization to investigate how $\sigma_{0}$ relates to both our measurement of $\sigma_{\mathrm{cl}}$ and our measure of $\sigma$ within $\approx r_{h}$, in a system with a constant-density core. ${ }^{6}$

In principle both $\sigma_{0}$ and $a$ are free parameters in Equation (4). The degree by which the scale radius $a$ differs from $r_{h}$ as determined from a surface-density or surface-brightness profile indicates how $M / L$ may vary with radius in the cluster (see, e.g., Lane et al. 2010b). Here, however, we again do not have a sufficiently large sample of kinematic tracers, especially at inner radii, to put adequate constraints on $a$. We therefore make the assumption that mass follows light (i.e., $a=r_{h}=34^{\prime \prime} .5$ ) and fit for $\sigma_{0}$. The results of this process are seen in Figure 10. We find $\sigma_{0}=2.52_{-0.18}^{+0.26} \mathrm{~km} \mathrm{~s}^{-1}$, only slightly larger than the value measured for our centralmost bin, but considerably in excess of our global dispersion.

\subsection{Cluster Mass and Luminosity}

Having estimated the central velocity dispersion of NGC 1846 we can in turn derive an estimate of its mass, since, for an isotropic Plummer model (see, e.g., Dejonghe 1987)

$$
M=\frac{64 a \sigma_{0}^{2}}{3 \pi G} .
$$

Substituting in our assumed value for $a=8.4 \mathrm{pc}$ and our estimate for $\sigma_{0}$, we obtain $M=\left(8.4_{-1.2}^{+1.7}\right) \times 10^{4} M_{\odot}$.

We would also like to obtain an estimate of the global mass-tolight ratio for NGC 1846. To do this we first need an expression for the cluster luminosity, which we derive using Equation (1) integrated with respect to $2 \pi r_{p} d r_{p}$ :

$$
L\left(r_{p}\right)=\pi r_{c}^{2} \Sigma_{V, 0}\left[\ln (\alpha)-4 \frac{\sqrt{\alpha}-1}{\sqrt{\beta}}+\frac{\alpha-1}{\beta}\right]
$$

where

$$
\alpha=1+\left(r_{p} / r_{c}\right)^{2} \text { and } \beta=1+\left(r_{t} / r_{c}\right)^{2}
$$

Here, $\Sigma_{V, 0}$ is the $V$-band surface brightness corresponding to $n_{0}$ in Equation (1). Note that in directly scaling the number-density

\footnotetext{
6 Most globular clusters are found to conform closely to models in which the density of stars within the cluster core is approximately constant. The family of King $(1962,1966)$ models are the most prominent examples, but others such as those of Elson et al. (1987) or Wilson (1975) are seen to provide superior fits in some cases (e.g., McLaughlin \& van der Marel 2005). The radial profile of Goudfrooij et al. (2009) for NGC 1846 clearly shows this cluster to be well described by models possessing constant-density cores.
}

profile of Goudfrooij et al. (2009) to a surface-brightness profile we assume that NGC 1846 is not strongly mass segregated. That it is a very diffuse cluster for its age (see, e.g., Mackey et al. 2008b; Keller et al. 2011) is consistent with this assertion. Note that we also made this assumption previously when setting $a=r_{h}$ in Equation (4) in order to estimate $\sigma_{0}$.

To obtain a measurement of the total cluster $V$-band luminosity $L_{V}$ we set $r_{p}=r_{t}$ in Equation (6) and estimated the central surface brightness $\Sigma_{V}(0)$ directly from our master ACS/WFC F555W reference image. To mitigate the effects of any miscentering, and random fluctuations due to the brightest stars, we measured the flux within a relatively large aperture of radius $r_{p}=10^{\prime \prime}$. This is acceptable because the core radius of NGC 1846 is even larger still. We obtained $\Sigma_{V}(0)=19.6 \pm 0.1$ mag $\operatorname{arcsec}^{-2}$, which, assuming that the LMC distance modulus is 18.5 and the foreground reddening $E(B-V)=0.08$, corresponds to $\Sigma_{V}(0)=670 \pm 62 L_{\odot} \mathrm{pc}^{-2}$. However, we must also take into account that NGC 1846 is set against a moderately dense LMC field-we measure the local surface brightness to be $\Sigma_{V, \mathrm{bkg}}=22.8 \mathrm{mag} \operatorname{arcsec}^{-2}=35 L_{\odot} \mathrm{pc}^{-2}$ by using regions on the ACS/WFC image beyond $r_{t}$. Thus, for the cluster only, $\Sigma_{V}(0)=635 \pm 62 L_{\odot} \mathrm{pc}^{-2}$ and, substituting into Equation (1), we find $\Sigma_{V, 0}=1.41 \Sigma_{V}(0)=897 \pm 87 L_{\odot} \mathrm{pc}^{-2}$. Then, from Equation (6), $L_{V}=(1.44 \pm 0.14) \times 10^{5} L_{\odot}$.

This leads to a global mass-to-light ratio $M / L_{V}=0.59_{-0.10}^{+0.13}$ for NGC 1846. To place this value in a useful context, we compare it to the predictions of two well-known population synthesis codes-the PÉGASE (v2.0) models of Fioc \& RoccaVolmerange (1997), and the GALAXEV models of Bruzual \& Charlot (2003) — for a cluster of the age and metal abundance of NGC 1846. For both sets of models we assume $Z=0.008$ and a single instantaneous burst of star formation. We further adopt the default initial stellar mass ranges $-0.1-120 M_{\odot}$ for PÉGASE and 0.1-100 $M_{\odot}$ for GALAXEV - and the closest available IMF to the Kroupa (2001) "universal” IMF. For the PÉGASE models this corresponds to the IMF of Kroupa et al. (1993) while for the Galaxev models it is the IMF of Chabrier (2003). The PÉGAsE models allow us to make the assumption that all white dwarfs are retained in the cluster, while all neutron stars and black holes are expelled upon formation. For the GALAXEV models we necessarily assume all stellar remnants remain in the cluster; the predicted mass-to-light ratios reported below would drop by a few hundredths if the contributions from neutron stars and black holes were excluded.

The results are as follows. The PÉGAsE models predict $M / L_{V}=0.69$ at an age of $1.6 \mathrm{Gyr}$ and $M / L_{V}=0.88$ at $2.0 \mathrm{Gyr}$, while the GALAXEV models predict $M / L_{V}=0.53$ and 0.70 at these two ages. The differences between the two sets of results seem to be mainly due to the assumed IMFs and initial stellar mass ranges. Taken at face value and within the precision of our present measurements, the global mass-tolight ratio we have observed for NGC 1846 agrees acceptably with expectations derived purely from analysis of its constituent stellar populations. Note, however, that the systemic rotation we have detected could well play an important enough role in the internal kinematics of the cluster that the use of Equation (5) when estimating $M / L_{V}$ may not be appropriate (see Sections 4.5 and 5).

\subsection{Relaxation Time}

Finally, we take the parameters measured above and use them to derive the central and half-mass relaxation times in NGC 1846. Following Djorgovski (1993), the central relaxation 
time is given by

$$
t_{r c}=8.338 \times 10^{6} \mathrm{yr} \times \frac{\rho_{0}^{1 / 2} r_{c}^{3}}{\bar{m} \ln \Lambda},
$$

where $\rho_{0}$ is the central mass density in the cluster, $\bar{m}$ is the mean stellar mass, and the units of mass and distance are solar masses and parsecs, respectively. The quantity $\ln \Lambda$ is the Coulomb logarithm where $\Lambda \approx 0.4 N$ if $N$ is the total number of stars in the system.

Simple population synthesis models (such as SIMCLUST; Deveikis et al. 2008) show that in a cluster of age $\approx 1.75 \mathrm{Gyr}$ with a Kroupa (2001) mass function, the mean stellar mass $\bar{m} \approx 0.5 M_{\odot}$. Here we again assume that NGC 1846 is not strongly mass segregated so that $\bar{m}$ does not vary significantly throughout the system. The number of stars in NGC 1846 is $N=M / \bar{m} \approx 2 M$. To derive $\rho_{0}$ we used the two procedures outlined by Djorgovski (1993) to convert our observed, extinction-corrected central surface-brightness $\Sigma_{V}(0)$ to a central luminosity density $j_{0}=71 \pm 5 L_{\odot} \mathrm{pc}^{-3}$, and then multiplied by our derived mass-to-light ratio so that $\rho_{0}=42_{-8}^{+10} M_{\odot} \mathrm{pc}^{-3}$. Substituting all these values into Equation (7) we obtain a central relaxation time $t_{r c}=2.4_{-0.2}^{+0.3} \mathrm{Gyr}$.

The half-mass, or median, relaxation time is given by (e.g., Binney \& Tremaine 1987)

$$
t_{r h}=2.055 \times 10^{6} \mathrm{yr} \times \frac{M^{1 / 2} r_{h}^{3 / 2}}{\bar{m} \ln \Lambda} .
$$

As before, the units of mass and distance in this equation are solar masses and parsecs, respectively. Application of the appropriate values for NGC 1846 yields $t_{r h}=2.6_{-0.2}^{+0.3} \mathrm{Gyr}$. This is only marginally longer than the central relaxation time, consistent with the observation that NGC 1846 is a diffuse cluster (i.e., the core radius $r_{c}$ is comparable to $r_{h}$ ).

\subsection{Robustness of the Kinematic Analysis}

The magnitude of the rotation that we infer in NGC 1846 is roughly comparable to the mean velocity dispersion in the system, which is quite unusual for a stellar cluster (see Section 5, below). Because of the potential significance of this observation, it is important to thoroughly assess the robustness of our kinematic analysis. There are two main reasons why it is necessary to undertake such a check. First, our sample of dynamical probes is comparatively small and so it is not inconceivable that, for example, a chance arrangement of velocity and position angle among a few stars could lead to a false rotation detection. Second, NGC 1846 has a non-negligible binary star fraction (note the strong binary star main sequences seen in the two CMDs in Figure 1) and so for a subset of stars in our ensemble the observed velocities likely possess extra components due to stellar companions rather than constituting a pure representation of the cluster kinematics.

To investigate these issues we developed 17 sets of $10^{5}$ random realizations of our measured sample, and calculated for each set (1) how frequently we detected a rotation signal comparable to that observed for NGC 1846; and (2) the mean ensemble velocity dispersion. We characterized a given set of models according to the assumed properties of its binary star population. This analysis is quite similar, at least in its principle, to that of McConnachie \& Côté (2010).

Each realization consisted of 22 targets at identical radii to our real sample, but at random position angles and with random velocities generated according to a well-defined set of rules. It could be argued that the radius assigned to each star should also be randomly selected, according to the density profile of NGC 1846. However, our measured sample is not solely defined by this density profile-rather it is the profile convolved with some complex and essentially unknown selection function for input to FLAMES (see Section 2.2). In the absence of this information, we felt the most sensible option was to maintain the radial distribution of the observed sample for each mock system.

For each target we first assigned a base velocity by selecting randomly from a Gaussian distribution of width specified by Equation (4) with $a=r_{h}=34.5$ and $\sigma_{0}=2.5 \mathrm{~km} \mathrm{~s}^{-1}$, evaluated at the appropriate radius. To this we added a random Gaussian deviate to represent the observational uncertainty, exactly as in Section 4.1. Next, we randomly assigned the star to be an unresolved binary, or not, according to the assumed binary fraction for the overall set to which the mock realization belonged. If a star was selected to be a binary we randomly generated a set of parameters to characterize the system, again according to the properties of the overall set, leading to an additional component to add to the base velocity. If, however, a star was not selected to be a binary, no further modification of its base velocity was made. Note that the planetary nebula Mo-17 was never selected to be a binary (since our velocity for this object comes from the nebula itself rather than the central star), but was otherwise treated identically to the other 21 stars in the sample.

Binary systems are characterized by the masses of the two components, $m_{1}$ and $m_{2}$ (where the mass of the primary $m_{1} \geqslant m_{2}$ ), the orbital period $P$, and the orbital eccentricity $e$. It is convenient to define the mass ratio $q=m_{2} / m_{1}$. The semi-major axis $a_{2}$ of the orbit of the secondary about the barycenter may be calculated using Kepler's third law, and the semi-major axis of the primary $a_{1}=q a_{2}$. To place a given binary system into the observational plane requires several additional parameters - the inclination $i$ of the orbit to the line of sight, the argument (or longitude) or periastron, $\omega$, and the orbital phase $\theta$ at which the observation was made. The radial velocity of the primary then varies as

$$
V_{b, 1}=\frac{2 \pi a_{1} \sin i}{P \sqrt{1-e^{2}}}[\cos (\theta+\omega)+e \cos (\omega)] .
$$

We defined each set of random realizations according to the distributions from which the mass ratio, period, and eccentricity of each binary were drawn. Mass ratios were selected from either a uniform distribution or the normal distribution of Duquennoy \& Mayor (1991), while periods were chosen according to either a uniform distribution in $\log P$ or the $\log$-normal distribution of Duquennoy \& Mayor (1991). Orbital eccentricities were selected from one of four distributions: circular orbits only $(e=0)$, a uniform distribution, the normal distribution of Duquennoy \& Mayor (1991), or the thermal distribution of Heggie (1975). The different combinations of these distributions defined 16 sets of models. We also computed a 17 th set with no binaries for comparison purposes.

Since all our observed targets are upper RGB stars (with a few possible AGB stars), for every mock binary system we set the primary mass $m_{1}=1.625 M_{\odot}$, based on the typical masses of upper RGB stars in the best-fitting Dartmouth isochrones (Dotter et al. 2008) for NGC 1846 (Goudfrooij et al. 2009). The binary fraction for a given set of models was defined using the observations of Milone et al. (2009) extrapolated according to 
Table 4

Results from the Mock Realizations of Our Measured Ensemble, including the Effects of Unresolved Binary Stars

\begin{tabular}{|c|c|c|c|c|c|c|c|c|c|c|c|c|c|c|}
\hline \multirow{2}{*}{$\begin{array}{l}\text { Set } \\
\text { Number }\end{array}$} & \multicolumn{4}{|c|}{ Assumed Distributions } & \multicolumn{3}{|c|}{ Peak $\geqslant 1.1 \mathrm{~km} \mathrm{~s}^{-1}(\%)$} & \multicolumn{3}{|c|}{ Peak $\geqslant 0.7 \mathrm{~km} \mathrm{~s}^{-1}(\%)$} & \multicolumn{4}{|c|}{ Mean Kinematics $\left(\mathrm{km} \mathrm{s}^{-1}\right)$} \\
\hline & $q$ & $\log P$ & $e$ & $f_{b}$ & $A_{\text {rot }}$ & $+\mathrm{rms}$ & $+R^{2}$ & $A_{\text {rot }}$ & $+\mathrm{rms}$ & $+R^{2}$ & $\bar{V}_{\text {sys }}$ & $\sigma_{V_{\text {sys }}}$ & $\bar{\sigma}_{\mathrm{cl}}$ & $\sigma_{\sigma_{\mathrm{cl}}}$ \\
\hline 01 & $\ldots$ & $\ldots$ & $\ldots$ & 0.00 & 2.01 & 0.23 & 0.39 & 8.88 & 1.31 & 0.97 & 239.10 & 0.41 & 1.79 & 0.32 \\
\hline 02 & Unif. & Unif. & Circ. & 0.33 & 6.28 & 0.33 & 0.72 & 18.15 & 1.35 & 1.30 & 239.11 & 0.51 & 2.18 & 0.42 \\
\hline 03 & Unif. & Unif. & Unif. & 0.33 & 5.98 & 0.34 & 0.74 & 17.80 & 1.34 & 1.23 & 239.09 & 0.50 & 2.18 & 0.42 \\
\hline 04 & Unif. & Unif. & Ther. & 0.33 & 5.93 & 0.32 & 0.75 & 17.66 & 1.32 & 1.26 & 239.11 & 0.50 & 2.16 & 0.43 \\
\hline 05 & Unif. & Unif. & Norm. & 0.33 & 6.20 & 0.33 & 0.73 & 17.96 & 1.26 & 1.23 & 239.09 & 0.50 & 2.18 & 0.42 \\
\hline 06 & Unif. & Norm. & Circ. & 0.33 & 6.01 & 0.33 & 0.69 & 18.10 & 1.35 & 1.26 & 239.11 & 0.50 & 2.16 & 0.42 \\
\hline 07 & Unif. & Norm. & Unif. & 0.33 & 5.98 & 0.31 & 0.67 & 17.58 & 1.38 & 1.26 & 239.10 & 0.50 & 2.17 & 0.42 \\
\hline 08 & Unif. & Norm. & Ther. & 0.33 & 5.86 & 0.38 & 0.76 & 17.44 & 1.30 & 1.25 & 239.10 & 0.50 & 2.16 & 0.41 \\
\hline 09 & Unif. & Norm. & Norm. & 0.33 & 5.76 & 0.31 & 0.75 & 17.61 & 1.29 & 1.25 & 239.10 & 0.50 & 2.17 & 0.42 \\
\hline 10 & Norm. & Unif. & Circ. & 0.55 & 8.21 & 0.32 & 0.90 & 21.73 & 1.21 & 1.40 & 239.10 & 0.53 & 2.33 & 0.43 \\
\hline 11 & Norm. & Unif. & Unif. & 0.55 & 8.24 & 0.33 & 0.86 & 21.68 & 1.25 & 1.34 & 239.09 & 0.53 & 2.33 & 0.43 \\
\hline 12 & Norm. & Unif. & Ther. & 0.55 & 8.07 & 0.33 & 0.89 & 21.58 & 1.21 & 1.38 & 239.10 & 0.54 & 2.33 & 0.43 \\
\hline 13 & Norm. & Unif. & Norm. & 0.55 & 8.23 & 0.38 & 0.88 & 21.72 & 1.25 & 1.33 & 239.10 & 0.54 & 2.32 & 0.42 \\
\hline 14 & Norm. & Norm. & Circ. & 0.55 & 7.96 & 0.34 & 0.81 & 21.14 & 1.24 & 1.30 & 239.09 & 0.54 & 2.30 & 0.42 \\
\hline 15 & Norm. & Norm. & Unif. & 0.55 & 7.78 & 0.32 & 0.89 & 21.19 & 1.26 & 1.40 & 239.10 & 0.52 & 2.29 & 0.43 \\
\hline 16 & Norm. & Norm. & Ther. & 0.55 & 7.80 & 0.36 & 0.90 & 20.96 & 1.28 & 1.39 & 239.09 & 0.53 & 2.31 & 0.42 \\
\hline 17 & Norm. & Norm. & Norm. & 0.55 & 7.79 & 0.29 & 0.80 & 20.93 & 1.22 & 1.31 & 239.10 & 0.53 & 2.29 & 0.42 \\
\hline
\end{tabular}

the assumed distribution of mass ratios. Milone et al. estimated the binary fractions for 12 intermediate-age LMC clusters from $H S T /$ ACS imaging by counting stars above the main sequence. Due to blends and photometric uncertainties they were only able to clearly identify binaries with $q \geqslant 0.6$ or 0.7 , depending on the quality of the imaging. They found typical binary fractions $f_{b} \sim 0.14$ for $q \geqslant 0.6$, or $f_{b} \sim 0.09$ for $q \geqslant 0.7$. For a uniform distribution in $q$ these correspond to a total binary fraction $f_{b} \approx 0.33$, while for the Duquennoy \& Mayor (1991) normal distribution they correspond to $f_{b} \approx 0.55$. We adopted these two values for our various sets of models, depending on the assumed distribution in $q$.

Having selected a given star to be a binary, our procedure for generating a radial velocity was as follows. We first randomly selected a mass ratio, period, and eccentricity from the relevant distributions. We defined a lower bound to allowed values of $q$ by noting that the mass limit for hydrogen-burning, $m_{2} \approx 0.08 \mathrm{M}_{\odot}$, corresponds to $q \approx 0.05$ in our case. We further defined limits to the allowed values of $P$ following the procedure outlined by McConnachie \& Côté (2010). Briefly, the lower limit is set by the minimum orbital separation before the onset of mass-transfer, while the upper limit is set by the orbital separation corresponding to the boundary between "hard" and "soft" binaries in NGC 1846 - the latter of which are efficiently destroyed by three- or four-body interactions within the cluster. Next, we determined the orientation of the binary by selecting the angles $i$ and $\omega$ from uniform distributions. Finally, we selected a number in the range $0-1$, also according to a uniform distribution, to represent the elapsed fraction of the binary's orbital period since periastron, and converted this into the phase $\theta$ by numerically solving Kepler's equation. With all the necessary parameters in hand, we determined the radial velocity according to Equation (9). To mimic our original identification of cluster members (Section 3.1), if the resulting velocity of the star was separated from the systemic cluster velocity by more than five times the velocity dispersion at that radius, we returned and regenerated all its binary parameters.

The results for all 17 sets of realizations are presented in Table 4. To illustrate our analysis procedure we consider set 1 - the control set with zero binary fraction. We quantified whether the inferred rotation of a mock system matched that observed for the real cluster, by using the amplitude of rotation and two quality of fit parameters. As described in Section 4.1, the rotation velocity implied by the NGC 1846 curve shown in the top panel of Figure 7 is $1.1 \pm 0.4 \mathrm{~km} \mathrm{~s}^{-1}$. The curve is, further, a high-quality sinusoid, suggesting that the inferred rotation is not simply due to stochastic deviations from zero at a few position angles. The rms residual for the best-fitting model is 0.27 , and the coefficient of determination $R^{2}=0.97$.

Just $2.01 \%$ of our mock systems in set 1 had inferred rotation of matching or greater amplitude than that measured for NGC 1846; however, only about a tenth of these $(0.23 \%$ of all systems) also had an rms residual value equal to or smaller than the best-fit model for NGC 1846. A slightly larger fraction $(0.39 \%$ of all systems) had matching or greater rotation amplitude together with an $R^{2}$ value equal to or greater than our best-fit model. Considering the lower bound on our NGC 1846 rotation amplitude of $\sim 0.7 \mathrm{~km} \mathrm{~s}^{-1}$, defined by the uncertainty on the measurement, the fraction of mock systems with matching or greater amplitude rose to $8.88 \%$. Again, however, only a small subset of these also had an equivalently high-quality rms value ( $1.31 \%$ of all systems) or $R^{2}$ value ( $0.97 \%$ of all systems).

To give a visual indication of the goodness-of-fit criteria, we show in Figure 11 several examples (taken from set 17, see below) where a rotation amplitude greater than that measured for NGC 1846 is inferred, but where the rms and/or the $R^{2}$ values indicate a poor-quality sinusoid. It is quite clear that these systems would not be mistaken for one in which a strong rotation signal had been reliably detected. We also show one example where stochastic fluctuations have resulted in a rotation curve that passes all our tests, and would have led to a false positive detection of rotation in the cluster. Fortunately, as outlined in Table 4, this is a rare occurrence.

For each random realization of our sample we also calculated the mean velocity dispersion using the maximum likelihood technique described previously. We then found the average dispersion across all random realizations in a given set, along with the standard deviation in this value. The mean systemic velocity for each random realization is a natural by-product of the maximum likelihood calculation, and for completeness we determined the average value for this quantity across each set as well. The results of this process are visible in Table 4. For 


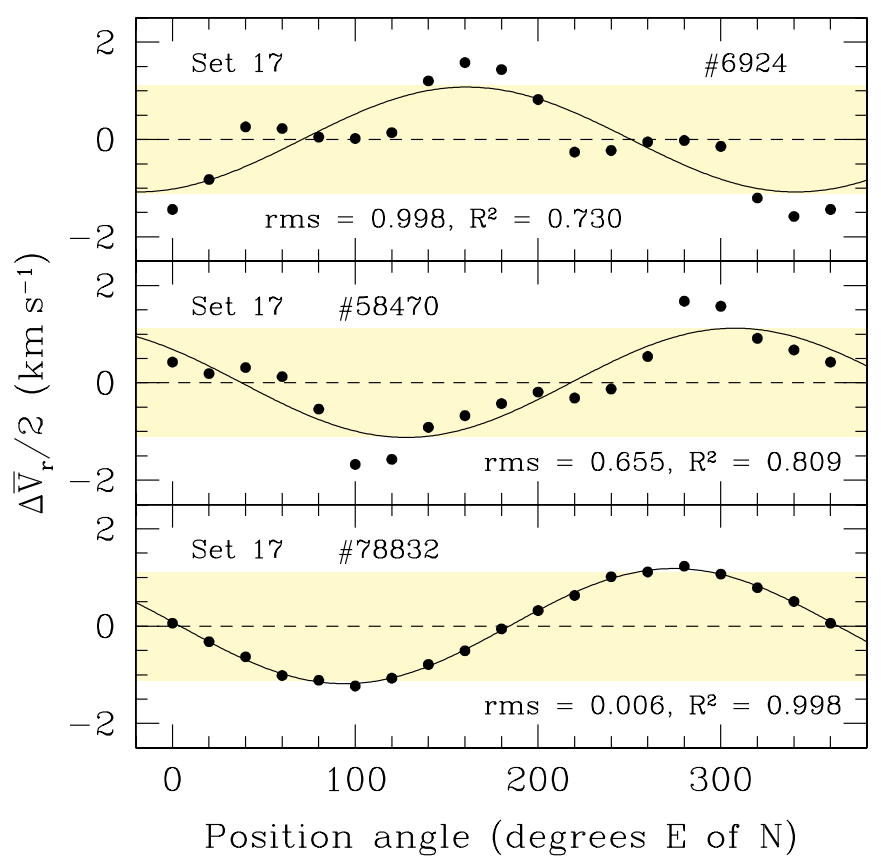

Figure 11. Example rotation curves for three mock systems in set 17 . We selected these to have comparable amplitudes to that measured for NGC 1846 (indicated, as previously, by the shaded region). The upper two panels show systems for which the best-fit models are of lower quality than for NGC 1846, as defined by the rms of the fit and the coefficient of determination $R^{2}$ (see the text). In the lower panel we show one rare example of a high-quality curve arising purely by chance. This particular realization had the best-quality fit of all $10^{5}$ mock systems in set 17 .

(A color version of this figure is available in the online journal.)

the control set 1 , we recover an average systemic velocity and velocity dispersion precisely matching those values derived for NGC 1846, including the uncertainties. This is to be expected, since the control run is the trivial case of re-measuring systems generated according to the velocity dispersion profile seen in Figure 10, with no contribution from binary stars.

We now consider the results for sets $2-17$, which possess binary population characteristics as outlined in Table 4. As expected, the additional velocity components due to the orbital motions of binary pairs do have some influence on our kinematic measurements. However, the effects do not appear to be strongly dependent on the adopted distributions for the orbital period or eccentricity. Changing the distribution for the mass ratio, $q$, has a more significant effect; however, this is most likely due to the resulting change in the inferred binary fraction in the cluster-more binaries imply a stronger influence on the kinematic measurements.

In terms of the inferred rotation, an increased percentage of the mock systems have amplitudes of matching or greater amplitude than our NGC 1846 measurement. For $f_{b}=0.33$ these values lie in the range $\sim 5.7 \%-6.3 \%$, while for $f_{b}=0.55$ they are higher, $\sim 7.7 \%-8.3 \%$. As before, however, only a very small subset of these systems possess high-quality sinusoidal rotation curves - the overall fractions are less than $1 \%$ for both goodness-of-fit criteria in all cases. Once again considering the conservative case defined by $A_{\text {rot }} \sim 0.7 \mathrm{~km} \mathrm{~s}^{-1}$, the percentages all rise; however, the overall fraction of systems in each set with a high-amplitude and high-quality rotation curve is again always close to or below $1.5 \%$.

The mean kinematics of each set of models are interesting. Binaries have no effect on the average systemic velocity, which always comes out at the measured NGC 1846 value. This is because the extra binary component of an individual radial velocity is equally likely to be pointing toward, or away from, the observer. However the binary stars do appreciably inflate the mean cluster velocity dispersion. In the case where $f_{b}=0.33$ the mean dispersion goes from $1.8 \mathrm{~km} \mathrm{~s}^{-1}$ to nearly $2.2 \mathrm{~km} \mathrm{~s}^{-1}$, while for $f_{b}=0.55$ the dispersion is typically just above $2.3 \mathrm{~km} \mathrm{~s}^{-1}$. As with the rotation measures, these values are not strongly dependent on the adopted distributions for the orbital period or eccentricity.

In summary, we draw the following conclusions from our Monte Carlo simulations. First, while it is not impossible for rotation signals comparable to that which we measured for NGC 1846 to arise stochastically, it is a very unusual occurrence with $<1 \%$ of systems in any given set exhibiting such characteristics. The presence of unresolved binary stars increases the number of systems for which a high amplitude of rotation could be inferred; however, the rotation curves for such systems would invariably not be mistaken for the expected sinusoidal variation of velocity with position angle. Thus, while we cannot rule out that the rotation we have observed in NGC 1846 is simply down to statistical fluke, we are better than $99 \%$ confident that it is a genuine signal.

Second, it is likely that our measured velocity dispersion for NGC 1846, $\sigma_{\mathrm{cl}}$, along with our inferred central velocity dispersion $\sigma_{0}$, are both inflated above their true values due to the presence of unresolved binary stars in our sample. In our simulations, an input value of $\sigma_{\mathrm{cl}}=1.81 \mathrm{~km} \mathrm{~s}^{-1}$ is increased to at most $\approx 2.33 \mathrm{~km} \mathrm{~s}^{-1}$. Since the various sources of dispersion add in quadrature, this implies the true $\sigma_{\mathrm{cl}}$ could be as low as $\approx 1.10 \mathrm{~km} \mathrm{~s}^{-1}$, and the true $\sigma_{0}$ as low as $\approx 2.05 \mathrm{~km} \mathrm{~s}^{-1}$. By comparison, our typical measurement uncertainty of $\approx 0.6 \mathrm{~km} \mathrm{~s}^{-1}$ on each individual velocity inflates the true dispersions by less than $0.1 \mathrm{~km} \mathrm{~s}^{-1}$ (note that this contribution is accounted for by the maximum likelihood technique that we employed to measure the cluster dispersion).

\section{DISCUSSION}

The key point of interest arising from our analysis of the internal kinematics of NGC 1846 concerns the degree of systemic rotation, which appears to be high compared with, for example, many Milky Way globular clusters, or indeed the few Magellanic Cloud clusters for which such measurements exist. We find a ratio $A_{\text {rot }} / \sigma_{0}=0.44 \pm 0.16$ if we adopt the mean amplitude of rotation $A_{\text {rot }}=1.1 \pm 0.4 \mathrm{~km} \mathrm{~s}^{-1}$. However, we also demonstrated that the maximum amplitude of rotation in NGC 1846 might well be as high as $\approx 2 \mathrm{~km} \mathrm{~s}^{-1}$ (especially since there is the additional unknown factor $\sin i$ required to deproject our rotation measurement-note that the mean value of $\sin i$ assuming a uniform distribution of inclination angles is $2 / \pi$ ). In this case, $A_{\text {rot }} / \sigma_{0} \sim 0.8$. We also showed that unresolved binary stars in our sample probably inflate the measured velocity dispersion by up to $\approx 0.5 \mathrm{~km} \mathrm{~s}^{-1}$, implying that the fraction of ordered motion with respect to pressure support is likely to be even larger still, $A_{\text {rot }} / \sigma_{0} \gtrsim 1.0$.

These latter two estimates are commensurate with the highest ratios measured in the sample of 24 Galactic globular clusters compiled by Bellazzini et al. (2012); indeed even our lower limit of $A_{\text {rot }} / \sigma_{0}=0.44$ would place NGC 1846 in the top $25 \%$ of this sample. They are also somewhat larger than the values measured for young Magellanic Cloud clusters. Fischer et al. (1992a) found $A_{\text {rot }} / \sigma_{0}=0.45 \pm 0.20$ in the $\approx 100 \mathrm{Myr}$ old cluster NGC 1866, while Fischer et al. (1993) measured a comparable 
degree of rotation in the $\approx 50 \mathrm{Myr}$ old cluster NGC 1850 . More recently, Hénault-Brunet et al. (2012a, 2012b) measured $A_{\text {rot }} / \sigma_{0}=0.60 \pm 0.30$ for the very young $(\approx 3 \mathrm{Myr})$ massive cluster R136 at the center of 30 Doradus. Notably, however, the only other intermediate-age LMC cluster investigated on a star-by-star basis, NGC 1978, shows no significant evidence for rotation (Fischer et al. 1992b).

When comparing the degree of internal rotation in NGC 1846 with rotation seen in Galactic globular clusters, it is important to bear in mind that NGC 1846 is still dynamically quite young. As demonstrated in Section 4.4, its central and median relaxation times are greater than the cluster age. It is possible, even likely, that these timescales were much shorter early on in the cluster's evolution (see, e.g., Mackey et al. 2007, 2008b); however, it is clear that in the ancient Galactic globular clusters any ordered motions have had far more opportunity to become randomized, thus suppressing $A_{\text {rot }} / \sigma_{0}$ relative to NGC 1846.

This is not the case for the young LMC clusters NGC 1850 and 1866, and R136, which, like NGC 1846, should be dynamically unevolved (e.g., Fischer et al. 1992a, 1993; Hénault-Brunet et al. 2012a). That the degree of rotation observed in NGC 1846 is, arguably, greater than in these young systems is a striking result. If, as we suspect, $A_{\text {rot }} / \sigma_{0} \approx 0.8-1.0$, then ordered rotation is comparable in importance to random motions in providing support against gravitational collapse. Following the discussion in Hénault-Brunet et al. (2012a), for this range in $A_{\text {rot }} / \sigma_{0}$, between $\approx 35 \%-45 \%$ of the total cluster kinetic energy would be in rotation.

NGC 1846 is only mildly elliptical—Goudfrooij et al. (2009) measure $\epsilon=0.12 \pm 0.02$. However, a cluster's ellipticity is not necessarily a good indication of its degree of internal rotation. Bellazzini et al. (2012) found no correlation between these two quantities in their sample of Galactic globular clusters. Furthermore, the lone globular cluster associated with the isolated Local Group dwarf irregular galaxy Wolf-Lundmark-Mellote shows no evidence for strong internal rotation despite displaying a high degree of ellipticity (Stephens et al. 2006). NGC 1978, for which Fischer et al. (1992b) found no evident signs of rotation, also has a very flattened shape with $\epsilon \approx 0.3$.

Bellazzini et al. (2012) demonstrated that for Galactic globular clusters $A_{\text {rot }} / \sigma_{0}$ correlates quite strongly with $[\mathrm{Fe} / \mathrm{H}]$, such that more metal-rich systems have stronger internal rotation. With $[\mathrm{Fe} / \mathrm{H}] \approx-0.4$, NGC 1846 fits this correlation well, although we again caution that we are comparing dynamically evolved systems with a dynamically young system. The suggestion made by Bellazzini et al. (2012) is that the observed correlation between $[\mathrm{Fe} / \mathrm{H}]$ and $A_{\text {rot }} / \sigma_{0}$ may hint that dissipative gas dynamics plays a significant role in the process of cluster formation, due to the fact that a larger metal content in a gas should imply a higher efficiency in energy dissipation via atomic transitions.

This idea is particularly relevant to the present case, where we strongly suspect the presence of multiple stellar generations in NGC 1846. Bekki $(2010,2011)$ uses hydrodynamic simulations to investigate the formation of a second generation of stars at the center of a globular cluster following the accretion of gas (including AGB ejecta) into its potential well (see also Bekki \& Mackey 2009). He finds that if the first generation of stars possesses even a very small net angular momentum, gaseous dissipation during accretion onto the cluster center leads to a dynamically cold, rapidly rotating second generation with $A_{\text {rot }} / \sigma_{0} \gtrsim 0.8$. The second generation is initially very centrally concentrated but this nested structure, and the strong rotational signature, ought to be smoothed out as a result of relaxation processes during the subsequent long-term evolution of the cluster. Since NGC 1846 is, dynamically speaking, still quite young, both the central concentration of the younger generation (as measured by Goudfrooij et al. 2009), and the rotational kinematics (as seen in the present work), are apparently still evident. Our possible detection of increasing rotation toward the cluster center is also consistent with this picture.

A more sensitive test of the model described above would be achieved if we could split our kinematic sample into earlier and later generations; however, to presage the results of our chemical abundance analysis somewhat (see Paper II), there is no obvious marker for achieving this - and in any case the present ensemble is probably too small for useful subdivision. The most reliable method would be to target a large number of stars across the EMSTO in order to directly correlate kinematics against position on the CMD and within the cluster. Such measurements, if at all possible, would require a considerable investment of telescope time but would provide critical information to test the different formation hypotheses.

Nonetheless, the fact that we have detected strong rotation in the first EMSTO cluster subjected to detailed dynamical examination is suggestive that this may be an important feature of these systems. In the future it will be critically important to enlarge the sample of intermediate-age Magellanic Cloud clusters for which internal kinematics have been measured, including both systems with and without an EMSTO. Realistic $\mathrm{N}$-body modeling of clusters such as those predicted by hydrodynamic simulations, in which there is a centrally concentrated, strongly rotating second generation of stars embedded in a more diffuse, pressure supported first generation, would also be extremely useful (although we recognize the current limitations placed on such models due to the maximum particle number of $\sim 1-2 \times 10^{5}$ ). An issue of particular interest is how the spatial and kinematic distinctions between the different generations propagate through the dynamical evolution of the system, especially where the cluster is strongly mass segregated at early times such that violent relaxation due to rapid stellar mass-loss drives significant expansion, as appears to be necessary for the intermediate-age EMSTO clusters (e.g., Keller et al. 2011).

\section{SUMMARY AND CONCLUSIONS}

In this paper we have described a detailed set of VLT/FLAMES observations of red giant stars in the peculiar intermediate-age LMC star cluster NGC 1846, along with the data reduction procedure we employed to extract and process individual spectra. In total, we targeted 29 stars within the nominal boundary of NGC 1846, of which 21 possess radial velocities indicating their membership of the cluster at high confidence. In addition, we targeted the planetary nebula Mo-17, and the radial velocity of this object indicates that it too is a member of the cluster.

We have used our spectra to investigate the elemental abundance patterns present in NGC 1846, including the possibility of star-to-star variations in light element abundances. These results will be presented in a forthcoming work (Paper II). In the present paper we took the radial velocity measurements for our sample and used these to conduct a thorough analysis of the internal kinematics of the cluster, with the following results:

1. NGC 1846 exhibits a significant degree of systemic rotation. The mean amplitude is $A_{\text {rot }}=1.1 \pm 0.4 \mathrm{~km} \mathrm{~s}^{-1}$, with the rotation axis oriented at $60 \pm 20^{\circ}$ east of north. 
There are indications that the rotation signal may vary with position in the cluster such that the amplitude increases toward the center and peaks somewhere within the half-light radius. The maximum amplitude may well be as high as $A_{\text {rot }} \approx 2 \mathrm{~km} \mathrm{~s}^{-1}$, especially considering that there is also a correction factor $\sin i$ for the unknown inclination of the rotation axis to the line of sight. An extensive suite of Monte Carlo models suggests that, because of the relatively small size of our sample and the presence of a significant population of unresolved binary stars in NGC 1846, stochastic fluctuations could reproduce the observed rotation curve; however, this only occurs very rarely-less than $\approx 0.3 \%$ of the time if no binaries are present, or less than $\approx 1 \%$ of the time for a cluster binary fraction of up to $f_{b}=0.55$.

2. We measure a mean velocity dispersion $\sigma_{\mathrm{cl}}=$ $1.81_{-0.29}^{+0.37} \mathrm{~km} \mathrm{~s}^{-1}$. Assuming a simple parameterization of the velocity dispersion fall-off with radius, the implied central velocity dispersion in the cluster is $\sigma_{0}=$ $2.52_{-0.18}^{+0.26} \mathrm{~km} \mathrm{~s}^{-1}$. Our Monte Carlo modeling suggests that the presence of unresolved binary stars in our sample could substantially inflate these quantities. If the binary fraction $f_{b}=0.55$, the true values could be as low as $\sigma_{\mathrm{cl}} \approx 1.1 \mathrm{~km} \mathrm{~s}^{-1}$ and $\sigma_{0} \approx 2.0 \mathrm{~km} \mathrm{~s}^{-1}$.

3 . The ratio of ordered motion to pressure support is formally $A_{\text {rot }} / \sigma_{0}=0.44 \pm 0.16$; however, accounting for the probable maximum amplitude of rotation in the cluster, the inclination factor $\sin i$, and the contribution of binary stars to inflating the observed velocity dispersion, this quantity is likely to be as high as $0.8-1.0$. In this case, between $\approx 35 \%-45 \%$ of the total cluster kinetic energy would be in rotation.

4. Under the assumption that mass follows light in the cluster, the mass of NGC 1846 is $\left(8.4_{-1.2}^{+1.7}\right) \times 10^{4} M_{\odot}$ and the implied mass-to-light ratio is $0.59_{-0.10}^{+0.13}$, consistent with predictions made purely on consideration of its constituent stellar populations. Note, however, that these quantities are derived assuming a "dispersion-only" cluster (Equation (5)). If internal rotation provides an important contribution against gravitational collapse, as seems probable, more sophisticated modeling will be required to obtain reliable estimates of mass and $M / L_{V}$.

5. The median relaxation time for NGC 1846 is $t_{r h}=$ $2.6_{-0.2}^{+0.3} \mathrm{Gyr}$, indicating that the cluster is dynamically youthful. Hence any kinematic signatures encoded during its formation ought to remain present.

The observation that substantial rotation is present in NGC 1846, at a magnitude comparable to that of the velocity dispersion, is consistent with the predictions of simulations modeling the formation of multiple generations in globular clusters (see, e.g., Bekki 2010, 2011). It would be of significant interest to improve our knowledge of the internal kinematics of this cluster by extending the present work to a much larger sample, ideally one in which the multiple generations could be easily identified. Similarly, by extending our analysis to additional intermediate-age Magellanic Cloud clusters, both with and without the EMSTO morphology, we could hope to learn whether strong internal rotation is a key signature of the formation of clusters with multiple constituent stellar populations.

We would like to thank the anonymous referee for their assistance in improving this paper, along with Mike Irwin and Vanessa Hill for helpful discussions at the commencement of the project. A.D.M. is grateful for financial support from the Australian Research Council in the form of an Australian Research Fellowship (Discovery Projects grant DP1093431). G.Da.C. is grateful for research support from the Australian Research Council through Discovery Projects grant DP120101237. A.M.N.F. and A.D.M. acknowledge support by a Marie Curie Excellence Grant from the European Commission under contract MCEXT-CT-2005-025869 during the early stages of this project.

This work is partially based on observations made with the NASA/ESA Hubble Space Telescope, obtained from the Data Archive at the Space Telescope Science Institute, which is operated by the Association of Universities for Research in Astronomy, Inc., under NASA contract NAS 5-26555. These observations are associated with programs 9891 and 10595.

This publication makes use of data products from the Two Micron All Sky Survey (2MASS), which is a joint project of the University of Massachusetts and the Infrared Processing and Analysis Center/California Institute of Technology, funded by the National Aeronautics and Space Administration and the National Science Foundation.

Facility: VLT:Kueyen (FLAMES/GIRAFFE).

\section{REFERENCES}

Anderson, J., Sarajedini, A., Bedin, L. R., et al. 2008, AJ, 135, 2055

Bastian, N., \& de Mink, S. E. 2009, MNRAS, 398, L11

Bedin, L. R., Piotto, G., Anderson, J., et al. 2004, ApJL, 605, 125

Bekki, K. 2010, ApJL, 724, 99

Bekki, K. 2011, MNRAS, 412, 2241

Bekki, K., Campbell, S. W., Lattanzio, J. C., \& Norris, J. E. 2007, MNRAS, 377,335

Bekki, K., \& Mackey, A. D. 2009, MNRAS, 394, 124

Bekki, K., \& Norris, J. E. 2006, ApJL, 637, 109

Bellazzini, M., Bragaglia, A., Carretta, E., et al. 2012, A\&A, 538, A18

Binney, J., \& Tremaine, S. 1987, Galactic Dynamics (Princeton, NJ: Princeton Univ. Press)

Bruzual, G., \& Charlot, S. 2003, MNRAS, 344, 1000

Carretta, E., Bragaglia, A., Gratton, R. G., et al. 2009a, A\&A, 505, 117

Carretta, E., Bragaglia, A., Gratton, R. G., et al. 2010a, ApJL, 714, 7

Carretta, E., Bragaglia, A., Gratton, R. G., et al. 2010b, A\&A, 520, 95

Carretta, E., Bragaglia, A., Gratton, R., D'Orazi, V., \& Lucatello, S. 2009b, A\&A, 508, 695

Carretta, E., Bragaglia, A., Gratton, R., \& Lucatello, S. 2009c, A\&A, 505, 139

Chabrier, G. 2003, PASP, 115, 763

Cohen, J. G., Kirby, E. N., Simon, J. D., \& Geha, M. 2010, ApJ, 725, 288

Conroy, C. 2012, ApJ, 758, 21

Conroy, C., \& Spergel, D. N. 2011, ApJ, 726, 36

Da Costa, G. S., Held, E. V., Saviane, I., \& Gullieuszik, M. 2009, ApJ, 705,1481

D’Antona, F., \& Caloi, V. 2004, ApJ, 611, 871

Decressin, T., Meynet, G., Charbonnel, C., Prantzos, N., \& Ekström, S. 2007, A\&A, 464, 1029

de Grijs, R., Gilmore, G. F., Mackey, A. D., et al. 2002, MNRAS, 337, 597

Dejonghe, H. 1987, MNRAS, 224, 13

D’Ercole, A., Vesperini, E., D’Antona, F., McMillan, S. L. W., \& Recchi, S. 2008, MNRAS, 391, 825

Deveikis, V., Narbutis, D., Stonkutè, R., Bridžius, A., \& Vansevičius, V. 2008, BaltA, 17, 351

Djorgovski, S. G. 1993, in ASP Conf. Ser. 50, Structure and Dynamics of Globular Clusters, ed. S. G. Djorgovski \& G. Meylan (San Francisco, CA: ASP), 373

Dolphin, A. E. 2000, PASP, 112, 1383

Dotter, A., Chaboyer, B., Jevremović, D., et al. 2008, ApJS, 178, 89

Dupree, A. K., Strader, J., \& Smith, G. H. 2011, ApJ, 728, 155

Duquennoy, A., \& Mayor, M. 1991, A\&A, 248, 485

Elson, R. A. W., Fall, S. M., \& Freeman, K. C. 1987, ApJ, 323, 54

Elson, R. A. W., Freeman, K. C., \& Lauer, T. R. 1989, ApJL, 347, 69

Ferraro, F. R., Dalessandro, E., Mucciarelli, A., et al. 2009, Natur, 462, 483

Fischer, P., Welch, D. L., Côté, P., Mateo, M., \& Madore, B. F. 1992a, AJ, 103,857 
Fischer, P., Welch, D. L., \& Mateo, M. 1992b, AJ, 104, 1086

Fischer, P., Welch, D. L., \& Mateo, M. 1993, AJ, 105, 938

Fioc, M., \& Rocca-Volmerange, B. 1997, A\&A, 326, 950

Geisler, D., Villanova, S., Carraro, G., et al. 2012, ApJL, 756, 40

Girardi, L., Eggenberger, P., \& Miglio, A. 2011, MNRAS, 412, L103

Girardi, L., Rubele, S., \& Kerber, L. 2009, MNRAS, 394, L74

Glatt, K., Grebel, E. K., Sabbi, E., et al. 2008, AJ, 136, 1703

Gott, R. J., III. 1973, ApJ, 186, 481

Goudfrooij, P., Puzia, T. H., Chandar, R., \& Kozhurina-Platais, V. 2011a, ApJ, 737,4

Goudfrooij, P., Puzia, T. H., Kozhurina-Platais, V., \& Chandar, R. 2009, AJ, 137,4988

Goudfrooij, P., Puzia, T. H., Kozhurina-Platais, V., \& Chandar, R. 2011b, ApJ, 737,3

Gratton, R., Sneden, C., \& Carretta, E. 2004, ARA\&A, 42, 385

Gratton, R. G., Carretta, E., \& Bragaglia, A. 2012, A\&ARv, 20, 50

Grocholski, A. J., Cole, A. A., Sarajedini, A., Geisler, D., \& Smith, V. V. 2006, AJ, 132, 1630

Harris, W. E. 1996, AJ, 112, 1487

Heggie, D. C. 1975, MNRAS, 173, 729

Hénault-Brunet, V., et al. 2012a, A\&A, 545, L1

Hénault-Brunet, V., et al. 2012b, A\&A, 546, A73

Hill, V., François, P., Spite, M., Primas, F., \& Spite, F. 2000, A\&A, 364, L19

Johnson, J. A., Ivans, I. I., \& Stetson, P. B. 2006, ApJ, 640, 801

Keller, S. C., Mackey, A. D., \& Da Costa, G. S. 2011, ApJ, 731, 22

King, I. 1962, AJ, 67, 471

King, I. R. 1966, AJ, 71, 64

Koekemoer, A. M., Fruchter, A. S., Hook, R. N., \& Hack, W. 2003, in 2002 HST Calibration Workshop, ed. S. Arribas, A. Koekemoer, \& B. Whitmore (Baltimore, MD: STScI), 337

Kontizas, M., Morgan, D. H., Kontizas, E., \& Dapergolas, A. 1996, A\&A, 307,359

Kroupa, P. 2001, MNRAS, 322, 231

Kroupa, P., Tout, C. A., \& Gilmore, G. F. 1993, MNRAS, 262, 545

Kupka, F., Piskunov, N. E., Ryabchikova, T. A., Stempels, H. C., \& Weiss, W. W. 1999, A\&AS, 138, 119

Lane, R. R., Kiss, L. L., Lewis, G. F., et al. 2009, MNRAS, 400, 917

Lane, R. R., Kiss, L. L., Lewis, G. F., et al. 2010a, MNRAS, 401, 2521

Lane, R. R., Kiss, L. L., Lewis, G. F., et al. 2010b, MNRAS, 406, 2732

Lardo, C., Bellazzini, M., Pancino, E., et al. 2011, A\&A, 525, 114

Letarte, B., Hill, V., Jablonka, P., et al. 2006, A\&A, 453, 547

Letarte, B., Hill, V., Tolstoy, E., et al. 2010, A\&A, 523, 17

Lynden-Bell, D. 1967, MNRAS, 136, 101

Mackey, A. D., \& Broby Nielsen, P. 2007, MNRAS, 379, 151

Mackey, A. D., Broby Nielsen, P., Ferguson, A. M. N., \& Richardson, J. C. 2008a, ApJL, 681, 17
Mackey, A. D., Broby Nielsen, P., Ferguson, A. M. N., \& Richardson, J. C. 2009, in IAU Symp. 256, The Magellanic System: Stars, Gas, and Galaxies, ed. J. Th. van Loon \& J. M. Oliveira (Cambridge: Cambridge Univ. Press), 305

Mackey, A. D., \& Gilmore, G. F. 2003a, MNRAS, 338, 85

Mackey, A. D., \& Gilmore, G. F. 2003b, MNRAS, 338, 120

Mackey, A. D., Wilkinson, M. I., Davies, M. B., \& Gilmore, G. F. 2007, MNRAS, 379, L40

Mackey, A. D., Wilkinson, M. I., Davies, M. B., \& Gilmore, G. F. 2008b, MNRAS, 386, 65

Martell, S. L., \& Grebel, E. K. 2010, A\&A, 519, 14

Martell, S. L., Smolinski, J. P., Beers, T. C., \& Grebel, E. K. 2011, A\&A, 534, 136

McConnachie, A. W., \& Côté, P. 2010, ApJL, 722, 209

McLaughlin, D. E., \& van der Marel, R. P. 2005, ApJS, 161, 304

Milone, A. P., Bedin, L. R., Piotto, G., et al. 2008, ApJ, 673, 241

Milone, A. P., Bedin, L. R., Piotto, G., \& Anderson, J. 2009, A\&A, 497, 755

Morgan, D. H. 1994, A\&AS, 103, 235

Mucciarelli, A., Carretta, E., Origlia, L., \& Ferraro, F. R. 2008, AJ, 136, 375

Mucciarelli, A., Bellazzini, M., Ibata, R., et al. 2012, MNRAS, 426, 2889

Mucciarelli, A., Origlia, L., Ferraro, F. R., \& Pancino, E. 2009, ApJL, 695, 134

Norris, J. E. 2004, ApJL, 612, 25

Olszewski, E. W., Schommer, R. A., Suntzeff, N. B., \& Harris, H. C. 1991, AJ, 101,515

Osterbrock, D. E., Fulbright, J. P., Martel, A. R., et al. 1996, PASP, 108, 277

Pasquini, L., Avila, G., Blecha, A., et al. 2002, Msngr, 110, 1

Pasquini, L., Mauas, P., Käufl, H. U., \& Cacciari, C. 2011, A\&A, 531, 35

Piotto, G., Bedin, L. R., Anderson, J., et al. 2007, ApJL, 661, 53

Piotto, G., Villanova, S., Bedin, L. R., et al. 2005, ApJ, 621, 777

Plummer, H. C. 1911, MNRAS, 71, 460

Pompéia, L., Hill, V., Spite, M., et al. 2008, A\&A, 480, 379

Reid, W. A., \& Parker, Q. A. 2006, MNRAS, 373, 521

Rubele, S., Girardi, L., Kozhurina-Platais, V., Goudfrooij, P., \& Kerber, L. 2011, MNRAS, 414, 2204

Rubele, S., Kerber, L., \& Girardi, L. 2010, MNRAS, 403, 1156

Shaw, R. A., Stanghellini, L., Villaver, E., \& Mutchler, M. 2006, ApJS, 167, 201

Sirianni, M., Jee, M. J., Benítez, N., et al. 2005, PASP, 117, 1049

Skrutskie, M. F., Cutri, R. M., Stiening, R., et al. 2006, AJ, 131, 1163

Stephens, A. W., Catelan, M., \& Contreras, R. P. 2006, AJ, 131, 1426

Stetson, P. B., \& Pancino, E. 2008, PASP, 120, 1332

Ventura, P., D’Antona, F., Mazzitelli, I., \& Gratton, R. 2001, ApJL, 550, 65

Villanova, S., Piotto, G., King, I. R., et al. 2007, ApJ, 663, 296

Walker, M. G., Mateo, M., Olszewski, E. W., et al. 2006, AJ, 131, 2114

Wilson, C. P. 1975, AJ, 80, 175

Yong, D., \& Grundahl, F. 2008, ApJL, 672, 29

Zaritsky, D., Harris, J., Thompson, I. B., \& Grebel, E. K. 2004, AJ, 128, 1606 